\title{
Massive MIMO Relay Systems with Multipair Wireless Information and Power Transfer
}

\author{
Hongwu Liu, ${ }^{1}$ Kyung Hi Chang, ${ }^{2}$ and Kyung Sup Kwak ${ }^{3}$ \\ ${ }^{1}$ Shandong Jiaotong University, Jinan 250357, China \\ ${ }^{2}$ Department of Electronics Engineering, Inha University, Incheon 22212, Republic of Korea \\ ${ }^{3}$ Department of Information and Communication Engineering, Inha University, Incheon 22212, Republic of Korea \\ Correspondence should be addressed to Kyung Sup Kwak; kskwak@inha.ac.kr
}

Received 25 November 2016; Accepted 5 February 2017; Published 26 February 2017

Academic Editor: Jeongyeup Paek

Copyright (C) 2017 Hongwu Liu et al. This is an open access article distributed under the Creative Commons Attribution License, which permits unrestricted use, distribution, and reproduction in any medium, provided the original work is properly cited.

\begin{abstract}
This paper investigates destination-aided simultaneous wireless information and power transfer (SWIPT) for a decode-and-forward relay network, in which massive multiple-input multiple-output antennas are deployed at relay to assist communications among multiple source-destination pairs. During relaying, energy signals are emitted from multiple destinations when multiple sources are sending their information signals to relay. With power splitting and unlimited antennas at relay, asymptotic expression of harvested energy is derived. The analysis reveals that asymptotic harvested energy is independent of fast fading effect of wireless channels; meanwhile transmission powers of each source and destination can be scaled down inversely proportional to the number of relay antennas. To significantly reduce energy leakage interference and multipair interference, zero-forcing processing and maximumratio combing/maximum-ratio transmission are employed at relay. Fundamental trade-off between harvested energy and achievable sum rate is quantified. It is shown that asymptotic sum rate is neither convex nor concave with respect to power splitting and destination transmission power. Thus, a one-dimensional embedded bisection algorithm is proposed to jointly determine the optimal power splitting and destination transmission power. It shows that destination-aided SWIPT are beneficial for harvesting energy and increasing sum rate. The significant sum rate improvements of the proposed schemes are verified by numerical results.
\end{abstract}

\section{Introduction}

Recently, simultaneous wireless information and power transfer (SWIPT) have been envisioned for wireless relay networks [1-3]. The focuses on SWIPT for relay networks mainly include two relaying protocols, that is, time switching (TS) relaying and power splitting (PS) relaying [1]. In TS relaying, a relay harvests energy from a source-emitted RF signal and forwards source signal in a time-division manner. In PS relaying, a relay extracts energy for forwarding from its received source signal with PS operation. Generally, PS relaying can increase its spectrum efficiency by reducing the consumed time slots compared to that of TS relaying. In attempting to effectively utilize available time slots, SWIPT schemes with destination-aided energy flow (EF) have emerged [2-4]. In [2], the destination-aided EF was proposed for TS relaying protocol. In [3], an autonomous multiple-input multipleoutput (MIMO) relay employing PS-based energy receiver was investigated with destination-aided EF. For cognitive relay systems, the authors in [4] proposed sending EFs from destination to energy-constrained relay.

In the above mentioned destination-aided EF transmission schemes [2,3], only a single source-destination pair was considered. However, the demands for relay-assisted SWIPT with multiple source-destination pairs have emerged. For instance, authors in [5] proposed several power allocation schemes for a relay network, where multiple source-destination pairs communicate with each other via a common energy harvesting relay. In [6], the outage performances of SWIPT in large-scale networks with/without relay were analyzed, where multiple receivers harvest energy using PS technique. By employing multiple energy harvesting relays to assist multipair communications, the authors in [7] proposed distributed PS for SWIPT by using game theory. In [8], multiple relays were applied to assist communications from multiple base stations to multiple cell-edge users and PS-based 
energy receivers were deployed at multiple relays. For wireless power transfer enabled collaborative mobile clouds, cellular data distributing among multiple mobile users was investigated in [9]. It has shown that the energy-constrained relays need to harvest energy from ambient radio-frequency (RF) signals to support multipair communications in future dense heterogeneous networks [6-10].

One challenge in relay-assisted SWIPT is a limited amount of harvested energy. Due to path-loss and inefficient RF-to-DC conversion, only a small fraction of energy emitted by a source can be harvested at relay. In order to harvest energy efficiently, smart antennas were employed in SWIPT systems (see [11] and references therein). In [12], TS and PS receivers were proposed for SWIPT in MIMO broadcasting channels. A low-complexity antenna switching for MIMO relay networks with SWIPT was investigated in [13]. Moreover, multiple antennas were deployed at relay to harvest energy from EF emitted by a single destination [3]. Although MIMO techniques have improved SWIPT performance to a certain extent, harvesting enough energy cannot be guaranteed by a limited number of antennas.

Recently, massive MIMO techniques were applied to improve wireless transmission capacity by exploiting its large array gain $[14,15]$. A review of massive MIMO can be found in [16]. By employing linear signal processing [17], massive MIMO techniques have obtained increased signal-tointerference-plus-noise ratio (SINR) and power efficiency, which makes massive MIMO suitable to be deployed in practical SWIPT prototypes [18]. In [19], massive MIMO antennas were employed at a hybrid data-and-energy access point such that the minimum data rate among user nodes can be maximized whenever the number of antennas at the access point grows without bound. In [20], the authors proposed a low-complexity antenna partitioning algorithm for SWIPT systems with massive MIMO. Subjecting to a minimum harvested energy constraint, interference can be mitigated and throughput can be maximized [20]. Note that all the above mentioned works on SWIPT with massive MIMO were constrained in single-hop communications systems. To the best of our knowledge, only a few works have been conducted for multipair massive MIMO relay networks with SWIPT; for example, the authors in [21] proposed TS and PS relaying protocols for multiway amplify-and-forward (AF) relay networks, where information sharing among multiple users was aided by a massive MIMO relay.

In this paper, we propose a two-phase relaying protocol with destination-aided EFs for a decode-and-forward (DF) relay network, where multiple source-destination pairs communicate via a massive MIMO relay [10]. Different from the work of [7], where synchronization among multiple relays is required, we consider only a single relay with massive MIMO antennas and destination-aided EFs. This model has many interests, for example, in environmental sensor networks, Internet of things, and future dense heterogeneous networks, where interuser communications can be realized with the aid of an energy-constrained relay [3-10]. The main contributions of this paper can be summarized as follows:

(i) The important performance metrics including asymptotic harvested energy and asymptotic achievable rate are derived for the case in which the number of relay antennas grows without bound.

(ii) We show that destination-aided EFs are beneficial for boosting energy harvesting and, hence, the achievable sum rate can be improved significantly with the aid of destination-aided EFs. The optimal destination transmission power that maximizes the achievable sum rate is derived in closed-form. The optimal PS factor that maximizes the achievable sum rate is derived in closed-form.

(iii) We also show that by using zero-forcing ( $\mathrm{ZF}$ ) processing and maximum-ratio combing/maximum-ratio transmission (MRC/MRT) at relay, energy leakage interference (ELI) and multipair interference (MI) can be cancelled completely as the number of relay antennas grows without bound. When the number of relay antennas is large, transmission powers of each source and each destination can be scaled inversely proportional to the number of relay antennas.

(iv) We show that asymptotic sum rate is neither convex nor concave with respect to PS and destination transmission power, such that conventional convex optimization methods cannot be applied. We propose a one-dimensional embedded bisection (EB) algorithm to jointly determine the optimal PS and destination transmission power, so that the sum rate can be improved significantly.

The rest of this paper is organized as follows. Section 2 describes the system model and presents ZF processing and MRC/MRT processing. Section 3 presents the asymptotic analysis of system performance. The one-dimensional EB algorithm is also presented in Section 3. Section 4 presents numerical results and discusses the system performance of the proposed schemes. Finally, Section 5 summarizes the contributions of this study.

Notation. The superscripts $(\cdot)^{T},(\cdot)^{*}$, and $(\cdot)^{H}$ represent the transpose, conjugate, and conjugate-transpose, respectively. $\mathbb{E}\{\cdot\}$ and $\operatorname{Var}(\cdot)$ stand for the expectation and variance operations, respectively. $\mathbb{C}^{M \times N}$ denotes the $M \times N$ complex space and $\mathscr{C} \mathscr{N}(\mathbf{0}, \boldsymbol{\Sigma})$ stands for the circularly symmetric complex Gaussian distribution with zero mean and variance matrix $\Sigma$. Unless otherwise stated, vectors and matrices are, respectively, represented by bold lowercase and uppercase letters. $\mathbf{I}_{K}$ is the $K \times K$ identity matrix, $\mathbf{0}_{M \times K}$ is the $M \times K$ zero matrix, and $\mathbf{0}_{K}$ is the $K \times K$ zero matrix. $\operatorname{Tr}(\boldsymbol{\Sigma})$ denotes trace operation of a matrix $\Sigma$.

\section{System Model}

A block diagram of the considered multipair massive MIMO DF relay network is depicted in Figure 1 , where $K$ singleantenna equipped sources $\left(\mathrm{S}_{1}, \mathrm{~S}_{2}, \ldots, \mathrm{S}_{K}\right)$ transmit their information signals to $K$ single-antenna equipped destinations $\left(\mathrm{D}_{1}, \mathrm{D}_{2}, \ldots, \mathrm{D}_{K}\right)$ via an $M$-antenna equipped wirelesspowered massive MIMO relay (R). Without loss of generality, we assume that $M$ is larger than $K$ in this paper. We also 


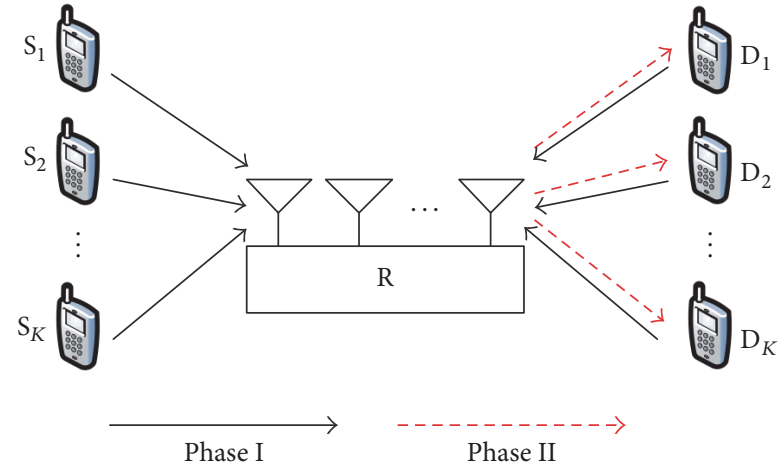

FIGURE 1: Multipair massive MIMO relay network.

assume that a direct link between the source and destination of each pair does not exist due to large path-loss or obstacles. All nodes work in half-duplex mode. Moreover, noises at relay's information detecting (ID) receiver and destinations' receivers are modeled as complex zero-mean additive noises, while noise power at relay's energy harvesting $(\mathrm{EH})$ receiver is assumed to be small, so that it is neglected.

The relaying protocol consists of two time phases. In phase I, all source nodes transmit their information signals $\mathrm{R}$ for forwarding. Meanwhile, all destination nodes transmit their EFs to R. Denote the PS factor by $\theta \in(0,1)$; the received signal power at $\mathrm{R}$ is split into a ratio of $\theta: 1-\theta$ for $\mathrm{EH}$ and ID processing, respectively. Denote the transmitted signals from the $K$ sources and $K$ destinations by $\mathbf{x}_{\mathrm{S}} \triangleq$ $\left[\begin{array}{llll}x_{\mathrm{S}, 1} & x_{\mathrm{S}, 2} & \cdots & x_{\mathrm{S}, K}\end{array}\right]^{T}$ and $\mathbf{x}_{\mathrm{D}} \triangleq\left[\begin{array}{llll}x_{\mathrm{D}, 1} & x_{\mathrm{D}, 2} & \cdots & x_{\mathrm{D}, \mathrm{K}}\end{array}\right]^{T}$, respectively, where $x_{\mathrm{S}, k}$ is the information signal transmitted by $\mathrm{S}_{k}$ and $x_{\mathrm{D}, k}$ is the EF signal transmitted by $\mathrm{D}_{k}$. We assume $\mathbb{E}\left\{\mathbf{x}_{\mathrm{S}} \mathbf{x}_{\mathrm{S}}^{H}\right\}=\mathbf{I}_{K}$ and $\mathbb{E}\left\{\mathbf{x}_{\mathrm{D}} \mathbf{x}_{\mathrm{D}}^{H}\right\}=\mathbf{I}_{K}$. The received signal at the input of the ID receiver can be written as

$$
\mathbf{y}_{\mathrm{R}, \mathrm{ID}}=(1-\theta)^{1 / 2}\left(\sqrt{P_{\mathrm{S}}} \mathbf{G}_{\mathrm{SR}} \mathbf{x}_{\mathrm{S}}+\sqrt{P_{\mathrm{D}}} \mathbf{G}_{\mathrm{DR}} \mathbf{x}_{\mathrm{D}}\right)+\mathbf{n}_{\mathrm{R}},
$$

where $\mathbf{G}_{\mathrm{SR}} \in \mathbb{C}^{M \times K}$ and $\mathbf{G}_{\mathrm{DR}} \in \mathbb{C}^{M \times K}$ are the channel matrices from the $K$ sources and $K$ destinations to R, respectively, $\sqrt{P_{\mathrm{S}}}$ and $\sqrt{P_{\mathrm{D}}}$ are the average transmission powers of each source and each destination, respectively, and $\mathbf{n}_{R}$ is the effective additive noise vector at $\mathrm{R}$ satisfying $\mathbb{E}\left\{\mathbf{n}_{\mathrm{R}} \mathbf{n}_{\mathrm{R}}^{H}\right\}=$ $\sigma_{\mathrm{R}}^{2} \mathbf{I}_{M}$. In practice, the effective noise can be defined as $\mathbf{n}_{\mathrm{R}} \triangleq$ $\sqrt{1-\theta} \mathbf{n}_{\mathrm{R}}^{\prime}+\mathbf{n}_{\mathrm{R}}^{\prime \prime}$, where $\mathbf{n}_{\mathrm{R}}^{\prime}$ and $\mathbf{n}_{\mathrm{R}}^{\prime \prime}$ are additive noise before and after the passive PS, respectively. The channel matrices $\mathbf{G}_{\mathrm{SR}}$ and $\mathbf{G}_{\mathrm{DR}}$ are, respectively, modeled as

$$
\begin{gathered}
\mathbf{G}_{\mathrm{SR}}=\mathbf{H}_{\mathrm{SR}} \mathbf{D}_{\mathrm{SR}}^{1 / 2}, \\
\mathbf{G}_{\mathrm{DR}}=\mathbf{H}_{\mathrm{DR}} \mathbf{D}_{\mathrm{DR}}^{1 / 2},
\end{gathered}
$$

where $\mathbf{H}_{\mathrm{SR}} \sim \mathscr{C} \mathcal{N}_{M \times K}\left(\mathbf{0}_{M \times K}, \mathbf{I}_{M} \otimes \mathbf{I}_{K}\right)$ and $\mathbf{H}_{\mathrm{DR}} \sim$ $\mathscr{C} \mathcal{N}_{M \times K}\left(\mathbf{0}_{M \times K}, \mathbf{I}_{M} \otimes \mathbf{I}_{K}\right)$ are the small-scale fading matrices modeling independent fast fading and $\mathbf{D}_{\mathrm{SR}}$ and $\mathbf{D}_{\mathrm{DR}}$ are the $K \times K$ diagonal matrices modeling the distance-related path-loss. The $k$ th diagonal elements of $\mathbf{D}_{\mathrm{SR}}$ and $\mathbf{D}_{\mathrm{DR}}$ are denoted by $\beta_{\mathrm{SR}, k}$ and $\beta_{\mathrm{DR}, k}$, respectively. Moreover, $\beta_{\mathrm{SR}, k}$ and $\beta_{\mathrm{DR}, k}$ are assumed to be constant over many channel coherence intervals as they change very slowly with time. Next, by exploiting reciprocity property of wireless channels, the channel matrix from $\mathrm{R}$ to the $K$ destinations in phase II can be defined as

$$
\mathbf{G}_{\mathrm{RD}}=\mathbf{G}_{\mathrm{DR}}^{T}=\mathbf{D}_{\mathrm{RD}}^{1 / 2} \mathbf{H}_{\mathrm{RD}},
$$

where $\mathbf{H}_{\mathrm{RD}}=\mathbf{H}_{\mathrm{DR}}^{T}$ and $\mathbf{D}_{\mathrm{RD}}=\mathbf{D}_{\mathrm{DR}}^{T}$.

At $\mathrm{R}$, the harvested energy at the $\mathrm{EH}$ receiver can be expressed as

$$
E_{\mathrm{h}}=\tau_{\mathrm{u}} \eta \theta\left(P_{\mathrm{S}} \operatorname{Tr}\left(\mathbf{G}_{\mathrm{SR}}^{H} \mathbf{G}_{\mathrm{SR}}\right)+P_{\mathrm{D}} \operatorname{Tr}\left(\mathbf{G}_{\mathrm{DR}}^{H} \mathbf{G}_{\mathrm{DR}}\right)\right),
$$

where $\tau_{\mathrm{u}}$ is the time duration of each phase and $\eta$ is the RFto-DC conversion efficiency. Thanks to the harvested energy in phase I, the relay transmission power $P_{\mathrm{R}}=E_{\mathrm{h}} / \tau_{\mathrm{u}}$ can be expressed as

$$
P_{\mathrm{R}}=\eta \theta\left(P_{\mathrm{S}} \operatorname{Tr}\left(\mathbf{G}_{\mathrm{SR}}^{H} \mathbf{G}_{\mathrm{SR}}\right)+P_{\mathrm{D}} \operatorname{Tr}\left(\mathbf{G}_{\mathrm{DR}}^{H} \mathbf{G}_{\mathrm{DR}}\right)\right) .
$$

In phase II, the relay forwards the decoded and processed signal, $\mathbf{x}_{\mathrm{R}} \in \mathbb{C}^{M \times 1}$, to the $K$ destinations by using the harvested energy during phase $I$. The received signals at the $K$ destinations can be written in a vector form as

$$
\mathbf{y}_{\mathrm{D}}=\sqrt{P_{\mathrm{R}}} \mathbf{G}_{\mathrm{RD}} \mathbf{x}_{\mathrm{R}}+\mathbf{n}_{\mathrm{D}},
$$

where $\mathbf{n}_{\mathrm{D}}$ is the additive noises at the $K$ destinations satisfying $\mathbb{E}\left\{\mathbf{n}_{\mathrm{D}} \mathbf{n}_{\mathrm{D}}^{T}\right\}=\sigma_{\mathrm{D}}^{2} \mathbf{I}_{K}$.

2.1. Channel Estimation. We adopt the minimum meansquared error (MMSE) method to obtain the channel state information (CSI) estimation. A part of coherence time is used for channel estimation. All the sources and destinations simultaneously send their pilot sequences of $\tau_{\mathrm{p}}$ symbols to $\mathrm{R}$. The received signal at $\mathrm{R}$ is given by

$$
\mathbf{Y}_{\mathrm{p}}=\sqrt{\tau_{\mathrm{p}} P_{\mathrm{p}}} \mathbf{G}_{\mathrm{SR}} \boldsymbol{\Phi}_{\mathrm{S}}+\sqrt{\tau_{\mathrm{p}} P_{\mathrm{p}}} \mathbf{G}_{\mathrm{DR}} \boldsymbol{\Phi}_{\mathrm{D}}+\mathbf{N}_{\mathrm{p}}
$$

where $P_{\mathrm{p}}$ is the transmission power of each symbol, $\mathbf{N}_{\mathrm{p}}$ is the additive noise matrix whose elements have zero mean and variance $\sigma_{\mathrm{R}, \mathrm{p}}^{2}$. In addition, the $k$ th rows of $\Phi_{\mathrm{S}} \in \mathbb{C}^{K \times \tau_{\mathrm{p}}}$ and $\Phi_{\mathrm{D}} \in \mathbb{C}^{K \times \tau_{\mathrm{p}}}$ are the pilot sequences transmitted from $\mathrm{S}_{k}$ and $\mathrm{D}_{k}$, respectively. It is required that $\tau_{\mathrm{p}} \geq 2 K$, so that the pairwise orthogonality of the pilot sequences, that is, $\boldsymbol{\Phi}_{\mathrm{S}} \boldsymbol{\Phi}_{\mathrm{S}}^{H}=$ $\mathbf{I}_{K}, \Phi_{\mathrm{D}} \boldsymbol{\Phi}_{\mathrm{D}}^{H}=\mathbf{I}_{K}$, and $\boldsymbol{\Phi}_{\mathrm{S}} \boldsymbol{\Phi}_{\mathrm{D}}^{H}=\mathbf{0}_{K}$, can be guaranteed.

The MMSE channel estimates of $\mathbf{G}_{\mathrm{SR}}$ and $\mathbf{G}_{\mathrm{RD}}$ are, respectively, given by [17]

$$
\begin{aligned}
& \widehat{\mathbf{G}}_{\mathrm{SR}}=\mathbf{G}_{\mathrm{SR}} \widetilde{\mathbf{D}}_{\mathrm{SR}}+\frac{1}{\sqrt{\tau_{\mathrm{p}} P_{\mathrm{p}}}} \mathbf{N}_{\mathrm{p}} \boldsymbol{\Phi}_{\mathrm{S}}^{H} \widetilde{\mathbf{D}}_{\mathrm{SR}}, \\
& \widehat{\mathbf{G}}_{\mathrm{DR}}=\mathbf{G}_{\mathrm{DR}} \widetilde{\mathbf{D}}_{\mathrm{DR}}+\frac{1}{\sqrt{\tau_{\mathrm{p}} P_{\mathrm{p}}}} \mathbf{N}_{\mathrm{p}} \boldsymbol{\Phi}_{\mathrm{D}}^{H} \widetilde{\mathbf{D}}_{\mathrm{DR}},
\end{aligned}
$$


where $\widetilde{\mathbf{D}}_{\mathrm{SR}} \triangleq\left(\left(\sigma_{\mathrm{R}, \mathrm{p}}^{2} \mathbf{D}_{\mathrm{SR}}^{-1} / \tau_{\mathrm{p}} P_{\mathrm{p}}\right)+\mathbf{I}_{K}\right)^{-1}$ and $\widetilde{\mathbf{D}}_{\mathrm{DR}} \triangleq\left(\left(\sigma_{\mathrm{R}, \mathrm{p}}^{2} \mathbf{D}_{\mathrm{DR}}^{-1} /\right.\right.$ $\left.\left.\tau_{\mathrm{p}} P_{\mathrm{p}}\right)+\mathbf{I}_{K}\right)^{-1}$. Denote the estimation error matrices of $\mathbf{G}_{\mathrm{SR}}$ and $\mathrm{G}_{\mathrm{DR}}$ by $\mathscr{E}_{\mathrm{SR}}$ and $\mathscr{E}_{\mathrm{SR}}$, respectively. Then, we have

$$
\begin{aligned}
\mathrm{G}_{\mathrm{SR}} & =\widehat{\mathbf{G}}_{\mathrm{SR}}+\mathscr{E}_{\mathrm{SR}}, \\
\mathrm{G}_{\mathrm{DR}} & =\widehat{\mathbf{G}}_{\mathrm{DR}}+\mathscr{E}_{\mathrm{DR}} .
\end{aligned}
$$

Since $\widehat{\mathbf{G}}_{\mathrm{SR}}, \mathscr{E}_{\mathrm{SR}}, \widehat{\mathbf{G}}_{\mathrm{DR}}$, and $\mathscr{E}_{\mathrm{DR}}$ are independent, we have $\widehat{\mathbf{G}}_{\mathrm{SR}} \sim \mathscr{C} \mathscr{N}\left(\mathbf{0}_{M \times K}, \widehat{\mathbf{D}}_{\mathrm{SR}}\right), \mathscr{E}_{\mathrm{SR}} \sim \mathscr{C} \mathscr{N}\left(\mathbf{0}_{M \times K}, \mathbf{D}_{\mathrm{SR}}-\widehat{\mathbf{D}}_{\mathrm{SR}}\right)$, $\widehat{\mathbf{G}}_{\mathrm{DR}} \sim \mathscr{C} \mathscr{N}\left(\mathbf{0}_{M \times K}, \widehat{\mathbf{D}}_{\mathrm{DR}}\right)$, and $\mathscr{E}_{\mathrm{DR}} \sim \mathscr{C} \mathscr{N}\left(\mathbf{0}_{M \times K}, \mathbf{D}_{\mathrm{DR}}-\right.$ $\widehat{\mathbf{D}}_{\mathrm{DR}}$ ), where $\widehat{\mathbf{D}}_{\mathrm{SR}}$ and $\widehat{\mathbf{D}}_{\mathrm{DR}}$ are diagonal matrices whose $k$ th elements are given by $\sigma_{\mathrm{SR}, k}^{2} \triangleq \tau_{\mathrm{p}} P_{\mathrm{p}} \beta_{\mathrm{SR}, k}^{2} /\left(\tau_{\mathrm{p}} P_{\mathrm{p}} \beta_{\mathrm{SR}, k}+\sigma_{\mathrm{R}, \mathrm{p}}^{2}\right)$ and $\sigma_{\mathrm{DR}, k}^{2} \triangleq \tau_{\mathrm{p}} P_{\mathrm{p}} \beta_{\mathrm{DR}, k}^{2} /\left(\tau_{\mathrm{p}} P_{\mathrm{p}} \beta_{\mathrm{DR}, k}+\sigma_{\mathrm{R}, \mathrm{p}}^{2}\right)$, respectively. Due to wireless channel reciprocity, $\widehat{\mathbf{G}}_{\mathrm{RD}}=\widehat{\mathbf{G}}_{\mathrm{DR}}^{T}$ is used as the MMSE channel estimate for $\mathbf{G}_{\mathrm{RD}}$.

2.2. Linear Processing at Relay. The relay employs linear processing for $\mathrm{DF}$ relaying. In phase $\mathrm{I}, \mathrm{R}$ uses a linear receiver matrix $\mathbf{W}^{H}$ to separate the received signal into $K$ streams. With the linear receiver, the received signal becomes

$$
\begin{aligned}
\mathbf{r}= & \mathbf{W}^{H} \mathbf{y}_{\mathrm{R}, \mathrm{ID}} \\
= & \sqrt{(1-\theta) P_{\mathrm{S}}} \mathbf{W}^{H} \mathbf{G}_{\mathrm{SR}} \mathbf{x}_{\mathrm{S}}+\sqrt{(1-\theta) P_{\mathrm{D}}} \mathbf{W}^{H} \mathbf{G}_{\mathrm{DR}} \mathbf{x}_{\mathrm{D}} \\
& +\mathbf{W}^{H} \mathbf{n}_{\mathrm{R}} .
\end{aligned}
$$

Then, the $k$ th element of $\mathbf{r}$ is used for detecting the signal transmitted from $S_{k}$, which can be expressed as

$$
\begin{aligned}
r_{k}= & \underbrace{\sqrt{(1-\theta) P_{\mathrm{S}}} \mathbf{w}_{k}^{H} \mathbf{g}_{\mathrm{SR}, k} x_{\mathrm{S}, k}}_{\text {desired signal }} \\
& +\underbrace{\sqrt{(1-\theta) P_{\mathrm{S}}} \sum_{j \neq k}^{K} \mathbf{w}_{k}^{H} \mathbf{g}_{\mathrm{SR}, j} x_{\mathrm{S}, j}}_{\text {multipair interference }} \\
& +\underbrace{\sqrt{(1-\theta) P_{\mathrm{D}} \mathbf{w}_{k}^{H} \mathbf{G}_{\mathrm{DR}} \mathbf{x}_{\mathrm{D}}}+\underbrace{\mathbf{w}_{k}^{H} \mathbf{n}_{\mathrm{R}}}_{\text {noise }}}_{\text {energy leakage interference }},
\end{aligned}
$$

where $\mathbf{w}_{k}$ and $\mathbf{g}_{\mathrm{SR}, k}$ are the $k$ th columns of $\mathbf{W}$ and $\mathbf{G}_{\mathrm{SR}}$, respectively.

After detecting the received signal, the relay node applies linear precoding to the detected signal before broadcasting. With DF relaying, the relay first decodes the original source signal and then regenerates the signal [22]. Thus, the transmitted signal at $\mathrm{R}$ can be expressed as

$$
\mathbf{x}_{\mathrm{R}}=\sqrt{P_{\mathrm{R}}} \mathbf{A} \mathbf{x}_{\mathrm{S}},
$$

where $\mathbf{A} \in \mathbb{C}^{M \times K}$ is a linear precoding matrix. From (6) and (12), the received signal at $D_{k}$ can be written as

$$
y_{\mathrm{D}, k}=\sqrt{P_{\mathrm{R}}} \mathbf{g}_{\mathrm{DR}, k}^{T} \mathbf{a}_{k} x_{\mathrm{S}, k}+\sqrt{P_{\mathrm{R}}} \sum_{j \neq k}^{K} \mathbf{g}_{\mathrm{DR}, k}^{T} \mathbf{a}_{j} x_{\mathrm{S}, j}+n_{\mathrm{D}, k},
$$

where $\mathbf{g}_{\mathrm{DR}, k}$ and $\mathbf{a}_{k}$ are the $k$ th columns of $\mathbf{G}_{\mathrm{DR}}$ and $\mathbf{A}$, respectively, and $n_{\mathrm{D}, k}$ is the $k$ th element of $\mathbf{n}_{\mathrm{D}}$.
2.2.1. ZF Processing. Since both the $K$ sources and $K$ destinations transmit their signals to $R$ in phase $I$ and $R$ broadcasts the processed streams to the $K$ destinations in phase II, MI will affect both the received signals at $\mathrm{R}$ and $K$ destinations. For the received signal at $\mathrm{R}, \mathrm{MI}$ is the term $\sqrt{(1-\theta) P_{\mathrm{S}}} \sum_{j \neq k}^{K} \mathbf{w}_{k}^{H} \mathbf{g}_{\mathrm{SR}, j} x_{\mathrm{S}, j}$, whereas, for the received signal at $\mathrm{D}_{k}, \mathrm{MI}$ is $\sqrt{P_{\mathrm{R}}} \sum_{j \neq k}^{K} \mathbf{g}_{\mathrm{DR}, k}^{T} \mathbf{a}_{j} x_{\mathrm{S}, j}$. Furthermore, the received signal at $\mathrm{R}$ also suffers from ELI resulting from EFs transmitted from the $K$ destinations to $\mathrm{R}$ in phase $\mathrm{I}$, which can be represented by $\sqrt{(1-\theta) P_{\mathrm{D}}} \mathbf{w}_{k}^{H} \mathbf{G}_{\mathrm{DR}} \mathbf{x}_{\mathrm{D}}$. In such a case, the relay applies the $\mathrm{ZF}$ receiver and $\mathrm{ZF}$ precoding to process the signal. By ZF processing, both MI and ELI are removed via projecting each intended information stream onto the orthogonal complement of MI and ELI. To this end, perfect CSI is required at R. However, when only the estimate of CSI is available at R, MI and ELI still exist.

The $\mathrm{ZF}$ processing matrices at $\mathrm{R}$ are given by

$$
\begin{aligned}
\mathbf{W}^{H} & =\mathbf{W}_{\mathrm{ZF}}^{H} \triangleq\left(\widehat{\mathbf{G}}_{\mathrm{SR}}^{H} \widehat{\mathbf{G}}_{\mathrm{SR}}\right)^{-1} \widehat{\mathbf{G}}_{\mathrm{SR}}^{H}, \\
\mathbf{A} & =\mathbf{A}_{\mathrm{ZF}} \triangleq \alpha_{\mathrm{ZF}} \widehat{\mathbf{G}}_{\mathrm{RD}}^{H}\left(\widehat{\mathbf{G}}_{\mathrm{RD}} \widehat{\mathbf{G}}_{\mathrm{RD}}^{H}\right)^{-1},
\end{aligned}
$$

respectively. In (15), the normalization factor $\alpha_{\mathrm{ZF}}$ is chosen to satisfy $\left|\mathbf{x}_{\mathrm{R}}\right|^{2}=P_{\mathrm{R}}$. Therefore, we have [23]

$$
\alpha_{\mathrm{ZF}} \triangleq \sqrt{\frac{1}{\operatorname{Tr}\left(\left(\widehat{\mathbf{G}}_{\mathrm{RD}}^{H} \widehat{\mathbf{G}}_{\mathrm{RD}}\right)^{-1}\right)}} .
$$

2.2.2. $M R C / M R T$ Processing. Since ZF processing neglects the noise effect, the corresponding detection performance is poor when the signal-to-noise ratio (SNR) is low. By contrast, MRC/MRT processing neglects $\mathrm{MI}$ to maximize the received SNR. As a result, MRC/MRT processing achieves a better detection performance than that of $\mathrm{ZF}$ processing in the low SNR region at the cost of achieving a worse detection performance than that of ZF processing in the high SNR region. Similarly to $\mathrm{ZF}$ processing, MRC/MRT processes the received signal and amplifies the processed signal according to (10) and (12). The MRC receiver and MRT precoding matrices can be, respectively, expressed as [23]

$$
\begin{aligned}
\mathbf{W}^{H} & =\mathbf{W}_{\mathrm{MRC}}^{H} \triangleq \widehat{\mathbf{G}}_{\mathrm{SR}}^{H}, \\
\mathbf{A} & =\mathbf{A}_{\mathrm{MRT}} \triangleq \alpha_{\mathrm{MRT}} \widehat{\mathbf{G}}_{\mathrm{RD}}^{H} .
\end{aligned}
$$

Furthermore, the normalization factor for the MRT precoding is given by [23]

$$
\alpha_{\mathrm{MRT}} \triangleq\left(\operatorname{Tr}\left(\widehat{\mathbf{G}}_{\mathrm{RD}}^{H} \widehat{\mathbf{G}}_{\mathrm{RD}}\right)\right)^{-1 / 2} .
$$

\section{Asymptotic Analysis}

In this section, the asymptotic harvested energy is derived for the case in which $M \rightarrow \infty$ and $K$ is fixed. The asymptotic symmetric sum rate over i.i.d. fading is derived for the case in which $M \rightarrow \infty, K \rightarrow \infty$, and $K / M$ is a constant. 
3.1. Asymptotic Harvested Energy Analysis. To begin with, the transmission powers at each source and each destination are, respectively, scaled inversely proportional to the number of antennas at $\mathrm{R}$, namely, $P_{\mathrm{S}}=E_{\mathrm{S}} / M$ and $P_{\mathrm{D}}=E_{\mathrm{D}} / M$, while keeping $E_{\mathrm{S}}$ and $E_{\mathrm{D}}$ fixed.

Proposition 1. Assume that the number of the sourcedestination pairs is fixed, the harvested energy at the relay as $M \rightarrow \infty$ is given by

$$
E_{h} \stackrel{\text { a.s. }}{\longrightarrow} \tau_{u} \eta \theta\left(E_{S} \operatorname{Tr}\left(\mathbf{D}_{S R}\right)+E_{D} \operatorname{Tr}\left(\mathbf{D}_{D R}\right)\right) \text {, }
$$

where $\stackrel{\text { a.s. }}{\longrightarrow}$ denotes almost sure convergence.

Proof. See Appendix A.

To obtain further insights, by letting $\mathbf{D}_{\mathrm{SR}}=\beta_{\mathrm{SR}} \mathbf{I}_{K}$ and $\mathbf{D}_{\mathrm{DR}}=\beta_{\mathrm{DR}} \mathbf{I}_{K}$, the harvested energy expression in (19) over i.i.d. fading can be asymptotically expressed as

$$
E_{\mathrm{h}}^{\infty}=\tau_{\mathrm{u}} \eta \theta K\left(\beta_{\mathrm{SR}} E_{\mathrm{S}}+\beta_{\mathrm{DR}} E_{\mathrm{D}}\right)
$$

Remark 2. The asymptotic harvested energy in (20) is independent of fast fading components of wireless channels. Furthermore, the asymptotic harvested energy increases with respect to the source and destination transmission powers, respectively. Therefore, for an expected value of the harvested energy at the relay, the source transmission power can be tuned down by tuning up the destination transmission power and vice versa. Moreover, since the array gain increases monotonically with respect to $M$, both the source and destination transmission powers can be scaled down inversely proportional to $M$ without any asymptotic performance degradation.

3.2. Using a Large Receive Antenna Array. To cancel ELI, orthogonal projection can be applied in detecting the desired signal. However, projecting ELI into its orthogonal space may contaminate the desired signal. Note that if the subspace spanned by ELI is orthogonal to the desired signal's subspace when $M$ is large, the orthogonal projection scheme can work well. Thanks to the massive array implementation, the channel vectors of the desired signal and ELI become nearly orthogonal when $M$ grows large. Therefore, we adopt the linear receiver as an orthogonal projection of the ELI. Consequently, ELI can be cancelled significantly by using the linear receiver with a large value of $M$. The main result is summarized in the following proposition.

Proposition 3. Assume that the number of source-destination pairs is fixed; the received signal at the relay for decoding the signal of the $k$ th source as $M \rightarrow \infty$ is given by

$$
\begin{gathered}
r_{k} \stackrel{\text { a.s. }}{\longrightarrow} \sqrt{(1-\theta) P_{S}} x_{S, k}, \quad \text { for ZF receiver }, \\
\frac{r_{k}}{M \sigma_{S R, k}^{2}} \stackrel{\text { a.s. }}{\longrightarrow} \sqrt{(1-\theta) P_{S}} x_{S, k}, \text { for MRC receiver. }
\end{gathered}
$$

Proof. See Appendix B.

Remark 4. The result in Proposition 3 shows that ELI and MI can be cancelled completely when $M$ grows to infinity. The received signal at the relay after using linear processing includes only the desired signal. Therefore, the capacity of $\mathrm{S}_{k} \rightarrow \mathrm{R}$ link grows without bound as $M$ approaches infinity.

\subsection{Using a Large Transmit Antenna Array}

Proposition 5. Assume that the number of source-destination pairs is fixed and the transmission power at the relay is supplied by the harvested energy, the received signal at $D_{k}$ for decoding the relayed information signal as $M \rightarrow \infty$ is given by

$$
\begin{aligned}
& \frac{y_{D, k}}{M \sqrt{M-K}} \\
& \stackrel{\text { a.s. }}{\longrightarrow} \sqrt{\eta \theta \sigma_{D R, k}^{4} \operatorname{Tr}\left(\widehat{\mathbf{D}}_{R D}^{-1}\right)\left(E_{S} \operatorname{Tr}\left(\mathbf{D}_{S R}\right)+E_{D} \operatorname{Tr}\left(\mathbf{D}_{D R}\right)\right)} x_{S, k}
\end{aligned}
$$

for ZF processing and

$$
\frac{y_{D, k}}{\sqrt{M}} \stackrel{\text { a.s. }}{\longrightarrow} \sqrt{\frac{\eta \theta \sigma_{D R, k}^{4}\left(E_{S} \operatorname{Tr}\left(\mathbf{D}_{S R}\right)+E_{D} \operatorname{Tr}\left(\mathbf{D}_{D R}\right)\right)}{\operatorname{Tr}\left(\widehat{\mathbf{D}}_{D R}\right)}} x_{S, k}
$$

\section{for MRC/MRT processing.}

Proof. See Appendix C.

To obtain further insights, the expressions in (23) and (24) of the received signal for decoding over i.i.d. fading can be, respectively, expressed as

$$
\begin{gathered}
\frac{y_{\mathrm{D}, k}}{M \sqrt{M-K}} \stackrel{\text { a.s. }}{\longrightarrow} \sqrt{\eta \theta K^{2} \sigma_{\mathrm{DR}}^{2}\left(\beta_{\mathrm{SR}} E_{\mathrm{S}}+\beta_{\mathrm{DR}} E_{\mathrm{D}}\right)} x_{\mathrm{S}, k}, \\
\frac{y_{\mathrm{D}, k}}{\sqrt{M}} \stackrel{\text { a.s. }}{\longrightarrow} \sqrt{\eta \theta \sigma_{\mathrm{DR}}^{2}\left(\beta_{\mathrm{SR}} E_{\mathrm{S}}+\beta_{\mathrm{DR}} E_{\mathrm{D}}\right)} x_{\mathrm{S}, k},
\end{gathered}
$$

where $\sigma_{\mathrm{DR}}^{2} \triangleq \tau_{\mathrm{p}} P_{\mathrm{p}} \beta_{\mathrm{DR}}^{2} /\left(\tau_{\mathrm{p}} P_{\mathrm{p}} \beta_{\mathrm{DR}}+\sigma_{\mathrm{R}, \mathrm{p}}^{2}\right)$.

Remark 6. By using linear processing at $\mathrm{R}$, the received signal at the destination $\mathrm{D}_{k}$ includes only the desired signal. Therefore, the capacity of $\mathrm{R} \rightarrow \mathrm{D}_{k}$ link grows without bound, which is similar to that of $\mathrm{S}_{k} \rightarrow \mathrm{R}$ link. Furthermore, we can see that the noise and MI disappeared when $M$ grows to infinity. It can be clearly seen from (25) that the EFs emitted by the destinations provide gain for decoding the information signal. Moreover, the received desired signals in (25) are independent of small-scale fading of wireless channels.

3.4. Sum Rate and Harvested Energy Trade-Off. The end-toend (e2e) achievable rate of the transmission link $\mathrm{S}_{k} \rightarrow \mathrm{R} \rightarrow$ $\mathrm{D}_{k}$ is given by

$$
R_{k}=\min \left\{R_{\mathrm{SR}, k}, R_{\mathrm{RD}, k}\right\},
$$


where $R_{\mathrm{SR}, k}$ and $R_{\mathrm{RD}, k}$ are the achievable rates of the transmission links $\mathrm{S}_{k} \rightarrow \mathrm{R}$ and $\mathrm{R} \rightarrow \mathrm{D}_{k}$, respectively. From (11), the received signal used for detecting $x_{\mathrm{S}, k}$ at the relay can be expressed as

$$
r_{k}=\underbrace{\sqrt{(1-\theta) P_{\mathrm{S}}} \mathbb{E}\left\{\widehat{\mathbf{g}}_{\mathrm{SR}, k}^{H} \mathbf{g}_{\mathrm{SR}, k}\right\} x_{\mathrm{S}, k}}_{\text {desired signal }}+\underbrace{\widetilde{n}_{\mathrm{R}, k}}_{\text {effective noise }},
$$

where the effective noise $\widetilde{n}_{\mathrm{R}, k}$ is given by

$$
\begin{aligned}
\widetilde{n}_{\mathrm{R}, k}= & \sqrt{(1-\theta) P_{\mathrm{S}}}\left(\widehat{\mathbf{g}}_{\mathrm{SR}, k}^{H} \mathbf{g}_{\mathrm{SR}, k}-\mathbb{E}\left\{\widehat{\mathbf{g}}_{\mathrm{SR}, k}^{H} \mathbf{g}_{\mathrm{SR}, k}\right\}\right) x_{\mathrm{S}, k} \\
& +\sqrt{(1-\theta) P_{\mathrm{S}}} \sum_{j \neq k}^{K} \widehat{\mathbf{g}}_{\mathrm{SR}, k}^{H} \mathbf{g}_{\mathrm{SR}, j} x_{\mathrm{S}, j} \\
& +\sqrt{(1-\theta) P_{\mathrm{D}}} \widehat{\mathbf{g}}_{\mathrm{SR}, k}^{H} \mathbf{G}_{\mathrm{DR}} \mathbf{x}_{\mathrm{D}}+\widehat{\mathbf{g}}_{\mathrm{SR}, k}^{H} \mathbf{n}_{\mathrm{R}} .
\end{aligned}
$$

It can be seen that the "desired signal" and "effective noise" in (27) are uncorrelated. Based on the fact that the worst-case uncorrelated additive noise is independent Gaussian noise of the same variance (see [17] and references therein), the achievable rate can be obtained as

$$
\begin{aligned}
R_{\mathrm{SR}, k} & =\log _{2}(1 \\
+ & \left.\frac{(1-\theta) P_{\mathrm{S}}\left|\mathbb{E}\left\{\mathbf{w}_{k}^{H} \mathbf{g}_{\mathrm{SR}, k}\right\}\right|^{2}}{(1-\theta) P_{\mathrm{S}} \operatorname{Var}\left(\mathbf{w}_{k}^{H} \mathbf{g}_{\mathrm{SR}, k}\right)+\mathrm{MI}_{k}+\mathrm{ELI}_{k}+\mathrm{AN}_{k}}\right),
\end{aligned}
$$

$$
\begin{aligned}
& R_{\text {sum }}=\mu K \\
& \quad \cdot \log _{2}\left(1+\min \left(\frac{(M-K)(1-\theta) P_{S} \sigma_{S R}^{2}}{K(1-\theta)\left(P_{S}\left(\beta_{S R}-\sigma_{S R}^{2}\right)+P_{D} \beta_{D R}\right)+\sigma_{R}^{2}}, \frac{\eta \theta M(M-K)\left(P_{S} \beta_{S R}+P_{D} \beta_{D R}\right)}{\eta \theta K M \sigma_{D R}^{-2}\left(P_{S} \beta_{S R}+P_{D} \beta_{D R}\right)\left(\beta_{D R}-\sigma_{D R}^{2}\right)+\sigma_{D}^{2} \sigma_{D R}^{-2}}\right)\right),
\end{aligned}
$$

where $\mathrm{MI}_{\mathrm{k}}, \mathrm{ELI}_{\mathrm{k}}$, and $\mathrm{AN}_{\mathrm{k}}$ represent the MI, ELI, and additive noise effect, respectively, and are, respectively, given by

$$
\begin{aligned}
& \mathrm{MI}_{k} \triangleq(1-\theta) P_{\mathrm{S}} \sum_{j \neq k}^{K} \mathbb{E}\left\{\left|\mathbf{w}_{k}^{H} \mathbf{g}_{\mathrm{SR}, j}\right|^{2}\right\}, \\
& \mathrm{ELI}_{k} \triangleq(1-\theta) P_{\mathrm{D}} \mathbb{E}\left\{\left\|\mathbf{w}_{k}^{H} \mathbf{G}_{\mathrm{DR}}\right\|^{2}\right\}, \\
& \mathrm{AN}_{k} \triangleq \mathbb{E}\left\{\left|\mathbf{w}_{k}^{H} \mathbf{n}_{\mathrm{R}}\right|^{2}\right\} .
\end{aligned}
$$

Similarly, considering (13), the achievable rate of the $\mathrm{R} \rightarrow \mathrm{D}_{k}$ link can be expressed as

$$
\begin{aligned}
& R_{\mathrm{RD}, k}=\log _{2}(1 \\
& \left.+\frac{\left|\mathbb{E}\left\{\mathbf{g}_{\mathrm{DR}, k}^{T} \mathbf{a}_{k}\right\}\right|^{2}}{\operatorname{Var}\left(\mathbf{g}_{\mathrm{DR}, k}^{T} \mathbf{a}_{k}\right)+\sum_{j \neq k}^{K} \mathbb{E}\left\{\left|\mathbf{g}_{\mathrm{DR}, k}^{T} \mathbf{a}_{j}\right|^{2}\right\}+\left(\sigma_{\mathrm{D}}^{2} / p_{\mathrm{R}}\right)}\right) .
\end{aligned}
$$

Although (33) implies that only statistical knowledge of the channel gains has been applied at $\mathrm{D}_{k}$ in decoding the information signal, the performance degradation compared to genie receiver is negligible for a large value of $M$ [17].

Theorem 7. For a finite number of relay antennas, the achievable rate of the system with $Z F$ processing over i.i.d. fading is given by where $\sigma_{S R}^{2} \triangleq \tau_{p} P_{p} \beta_{S R}^{2} /\left(\tau_{p} P_{p} \beta_{S R}+\sigma_{R, p}^{2}\right), \sigma_{D R}^{2} \triangleq \tau_{p} P_{p} \beta_{D R}^{2} /$ $\left(\tau_{p} P_{p} \beta_{D R}+\sigma_{R, p}^{2}\right)$, and the pre-log factor $\mu \triangleq \tau_{u} /\left(2 \tau_{u}+\tau_{p}\right)$ resulted from the pilot transmission and half-duplex relaying.

Proof. See Appendix D.

Remark 8. We can see from (34) that the achievable rates of the dual-hop links grow without bound as $M \rightarrow \infty$.
Therefore, the e2e performance can be always improved by using a more large antenna array when $\mathrm{ZF}$ processing is employed. When $M \rightarrow \infty, K \rightarrow \infty$, and $K / M$ is a constant, the asymptotic sum rate over i.i.d. fading becomes

$$
R_{\text {sum }}^{\infty}=\mu K \log _{2}\left(1+\min \left(\gamma_{\mathrm{SR}}, \gamma_{\mathrm{RD}}\right)\right),
$$

where

$$
\begin{aligned}
& \gamma_{\mathrm{SR}} \triangleq \frac{(1-\theta) E_{\mathrm{S}} \sigma_{\mathrm{SR}}^{2}}{(1 /(M / K-1))(1-\theta)\left(E_{\mathrm{S}}\left(\beta_{\mathrm{SR}}-\sigma_{\mathrm{SR}}^{2}\right)+E_{\mathrm{D}} \beta_{\mathrm{DR}}\right)+1 /(1-K / M) \sigma_{\mathrm{R}}^{2}}, \\
& \gamma_{\mathrm{RD}} \triangleq \frac{\eta \theta(M-K)\left(E_{\mathrm{S}} \beta_{\mathrm{SR}}+E_{\mathrm{D}} \beta_{\mathrm{DR}}\right)}{\eta \theta K \sigma_{\mathrm{DR}}^{-2}\left(E_{\mathrm{S}} \beta_{\mathrm{SR}}+E_{\mathrm{D}} \beta_{\mathrm{DR}}\right)\left(\beta_{\mathrm{DR}}-\sigma_{\mathrm{DR}}^{2}\right)+\sigma_{\mathrm{D}}^{2} \sigma_{\mathrm{DR}}^{-2}}
\end{aligned}
$$


denote the asymptotic SINRs of the first-hop and second-hop for each source-destination pair, respectively. This expression shows that, although the SINR of the $S_{k} \rightarrow$ R link is decreased by ELI, the SINR of the $\mathrm{R} \rightarrow \mathrm{D}_{k}$ link can be improved by employing destination-aided EFs. Therefore, the e2e performance can be improved when it is limited by the $\mathrm{R} \rightarrow \mathrm{D}_{k}$ link. Furthermore, we can reduce the transmission power of each source and each destination proportional to $1 / M$, meanwhile maintaining a given quality-of-service.

Proposition 9. With ZF processing, the asymptotic sum rate is neither convex nor concave with respect to destination transmission power and the optimal destination transmission power that achieves the allowable maximum asymptotic sum rate is given by

$$
E_{D}^{Z F}= \begin{cases}0, & E_{S} \geq \frac{E_{Z F}}{\beta_{S R}} \\ \frac{E_{Z F}-E_{S} \beta_{S R}}{\beta_{D R}}, & E_{S}<\frac{E_{Z F}}{\beta_{S R}},\end{cases}
$$

where $E_{Z F} \triangleq\left(-b_{1}+c_{1}+\sqrt{\left(b_{1}-c_{1}\right)^{2}+4 a_{1} d_{1}}\right) / 2 a_{1}$ with $a_{1} \triangleq$ $K /(M-K) E_{S} \sigma_{S R}^{2}, b_{1} \triangleq M \sigma_{R}^{2} /(M-K)(1-\theta) E_{S} \sigma_{S R}^{2}-K /(M-K)$, $c_{1} \triangleq K \sigma_{D R}^{-2}\left(\beta_{D R}-\sigma_{D R}^{2}\right) /(M-K)$, and $d_{1} \triangleq \sigma_{D}^{2} \sigma_{D R}^{-2} / \eta \theta(M-K)$.

Proof. See Appendix E.

Proposition 10. With $Z F$ processing, the asymptotic sum rate is concave with respect to $\theta$ and the optimal $\theta$ that achieves the allowable maximum asymptotic sum rate is given by

$$
=\frac{a_{2}+b_{2}-c_{2}+d_{2}-\sqrt{\left(a_{2}+b_{2}-c_{2}\right)^{2}-2\left(a_{2}-b_{2}-c_{2}\right) d_{2}+d_{2}^{2}}}{2\left(a_{2}-c_{2}\right)},
$$

where $a_{2} \triangleq K\left(E_{S}\left(\beta_{S R}-\sigma_{S R}^{2}\right)+E_{D} \beta_{D R}\right) /(M-K) E_{S} \sigma_{S R}^{2}, b_{2} \triangleq$ $M \sigma_{R}^{2} /(M-K) E_{S} \sigma_{S R}^{2}, c_{2} \triangleq K \sigma_{D R}^{-2}\left(\beta_{D R}-\sigma_{D R}^{2}\right) /(M-K)$, and $d_{2} \triangleq \sigma_{D}^{2} \sigma_{D R}^{-2} / \eta(M-K)\left(E_{S} \beta_{S R}+E_{D} \beta_{D R}\right)$.

Proof. See Appendix F.
Theorem 11. With ZF processing, the asymptotic sum rate is neither convex nor concave with respect to $\left(\theta, E_{D}\right)$ and there is a unique optimal $\left(\theta^{*}, E_{D}^{*}\right)$ that achieves the allowable maximum asymptotic sum rate.

Proof. See Appendix G.

Since the asymptotic sum rate is neither convex nor concave with respect to $\left(\theta, E_{\mathrm{D}}\right)$, the optimal $\left(\theta^{*}, E_{\mathrm{D}}^{*}\right)$ cannot be obtained by applying conventional convex optimization methods [24]. However, Theorem 11 shows that the optimal $\left(\theta^{*}, E_{\mathrm{D}}^{*}\right)$ is unique and Proposition 10 shows that the asymptotic sum rate is concave with respect to $\theta$, whereas Proposition 9 shows that for any given $\theta$ the corresponding optimal $E_{\mathrm{D}}^{\mathrm{ZF}}$ is uniquely determined. Thus, the twodimensional searching for the optimal $\left(\theta^{*}, E_{\mathrm{D}}^{*}\right)$ can proceed with a one-dimensional concave optimizing for $\theta^{*}$ with the corresponding $E_{\mathrm{D}}^{*}$ explicitly determined by (38). Since bisection is effective in searching optimal solution of a concave function [24], this paper proposes a one-dimensional $\mathrm{EB}$ algorithm to search the optimal $\left(\theta^{*}, E_{\mathrm{D}}^{*}\right)$ with noting that the optimal $E_{\mathrm{D}}^{*}$ is embedded in one-dimensional bisection. The proposed EB algorithm is presented in Algorithm 1.

To quantify the trade-off between the harvested energy and achievable sum rate, we solve for $\theta$ in (35) and substitute it into (20). The trade-off between the asymptotic harvested energy and sum rate with $\mathrm{ZF}$ processing can be derived as

$$
\begin{aligned}
E_{\mathrm{h}}^{\infty}= & \tau_{\mathrm{u}} \eta K\left(\beta_{\mathrm{SR}} E_{\mathrm{S}}+\beta_{\mathrm{RD}} E_{\mathrm{D}}\right) \\
& \times \begin{cases}\frac{d_{2} \Lambda}{c_{2} \Lambda+1}, & \theta<\theta^{\mathrm{ZF}} \\
1+\frac{b_{2} \Lambda}{a_{2} \Lambda-1}, & \theta \geq \theta^{\mathrm{ZF}},\end{cases}
\end{aligned}
$$

where $a_{2}, b_{2}, c_{2}$, and $d_{2}$ are the same as those of Proposition 10 and

$$
\Lambda \triangleq 2^{R_{\text {sum }}^{\infty} / \mu K}-1
$$

Theorem 12. For a finite number of relay antennas, the achievable rate of the system with MRC/MRT processing over i.i.d. fading is given by

$$
R_{\text {sum }}=\mu K \log _{2}\left(1+\min \left(\frac{M(1-\theta) P_{S} \sigma_{S R}^{2}}{K(1-\theta)\left(P_{S} \beta_{S R}+P_{D} \beta_{D R}\right)+\sigma_{R}^{2}}, \frac{M^{2} \eta \theta \sigma_{D R}^{2}\left(P_{S} \beta_{S R}+P_{D} \beta_{D R}\right)}{K M \eta \theta \beta_{D R}\left(P_{S} \beta_{S R}+P_{D} \beta_{D R}\right)+\sigma_{D}^{2}}\right)\right) .
$$

Proof. See Appendix H.

Remark 13. We can see from (42) that, as $M \rightarrow \infty$, the achievable rates of the dual-hop links grow without bound.
Thus, the e2e performance can be always improved by using a more large antenna array when MRC/MRT processing is employed. When $M \rightarrow \infty, K \rightarrow \infty$, and $K / M$ is constant, the asymptotic symmetric sum rate over i.i.d. fading is given by

$$
R_{\mathrm{sum}}^{\infty}=\mu K \log _{2}\left(1+\min \left(\frac{(1-\theta) E_{\mathrm{S}} \sigma_{\mathrm{SR}}^{2}}{(1-\theta)\left(E_{\mathrm{S}} \beta_{\mathrm{SR}}+E_{\mathrm{D}} \beta_{\mathrm{DR}}\right) K / M+\sigma_{\mathrm{R}}^{2}}, \frac{M \eta \theta \sigma_{\mathrm{DR}}^{2}\left(E_{\mathrm{S}} \beta_{\mathrm{SR}}+E_{\mathrm{D}} \beta_{\mathrm{DR}}\right)}{K \eta \theta \beta_{\mathrm{DR}}\left(E_{\mathrm{S}} \beta_{\mathrm{SR}}+E_{\mathrm{D}} \beta_{\mathrm{DR}}\right)+\sigma_{\mathrm{D}}^{2}}\right)\right) .
$$


(1) Initialize $\left(\theta_{a}, \theta_{b}\right) \subset(0,1)$ and $\varepsilon$.

(2) If $\theta_{b}-\theta_{a}>\varepsilon$, go to step (3), otherwise go to step (6).

(3) Set $\theta_{c}=\left(\theta_{a}+\theta_{b}\right) / 2, \theta_{a}^{\prime}=\left(\theta_{a}+\theta_{c}\right) / 2$, and $\theta_{b}^{\prime}=\left(\theta_{b}+\theta_{c}\right) / 2$. Compute $E_{\mathrm{D}}^{\mathrm{ZF}}\left(\theta_{a}^{\prime}\right), E_{\mathrm{D}}^{\mathrm{ZF}}\left(\theta_{b}^{\prime}\right)$, and $E_{\mathrm{D}}^{\mathrm{ZF}}\left(\theta_{c}^{\prime}\right)$ according to $(38)$;

Compute $R_{\text {sum }}^{\infty}\left(\theta_{a}^{\prime}, E_{\mathrm{D}}^{\mathrm{ZF}}\left(\theta_{a}^{\prime}\right)\right), R_{\text {sum }}^{\infty}\left(\theta_{b}^{\prime}, E_{\mathrm{D}}^{\mathrm{ZF}}\left(\theta_{b}^{\prime}\right)\right)$, and $R_{\text {sum }}^{\infty}\left(\theta_{c}, E_{\mathrm{D}}^{\mathrm{ZF}}\left(\theta_{c}\right)\right)$ according to (35).

(4) If $R_{\mathrm{sum}}^{\infty}\left(\theta_{a}^{\prime}, E_{\mathrm{D}}^{\mathrm{ZF}}\left(\theta_{a}^{\prime}\right)\right) \leq R_{\mathrm{sum}}^{\infty}\left(\theta_{c}, E_{\mathrm{D}}^{\mathrm{ZF}}\left(\theta_{c}\right)\right) \leq R_{\mathrm{sum}}^{\infty}\left(\theta_{b}^{\prime}, E_{\mathrm{D}}^{\mathrm{ZF}}\left(\theta_{b}^{\prime}\right)\right)$, set $\theta_{a}=\theta_{a}^{\prime}$; If $R_{\text {sum }}^{\infty}\left(\theta_{a}^{\prime}, E_{\mathrm{D}}^{\mathrm{ZF}}\left(\theta_{a}^{\prime}\right)\right) \geq R_{\text {sum }}^{\infty}\left(\theta_{c}, E_{\mathrm{D}}^{\mathrm{ZF}}\left(\theta_{c}\right)\right) \geq R_{\text {sum }}^{\infty}\left(\theta_{b}^{\prime}, E_{\mathrm{D}}^{\mathrm{ZF}}\left(\theta_{b}^{\prime}\right)\right)$, set $\theta_{b}=\theta_{b}^{\prime}$; If $R_{\text {sum }}^{\infty}\left(\theta_{c}, E_{\mathrm{D}}^{\mathrm{ZF}}\left(\theta_{c}\right)\right) \geq \max \left(R_{\text {sum }}^{\infty}\left(\theta_{a}^{\prime}, E_{\mathrm{D}}^{\mathrm{ZF}}\left(\theta_{a}^{\prime}\right)\right), R_{\text {sum }}^{\infty}\left(\theta_{b}^{\prime}, E_{\mathrm{D}}^{\mathrm{ZF}}\left(\theta_{b}^{\prime}\right)\right)\right)$, set $\theta_{a}=\theta_{a}^{\prime}$ and $\theta_{b}=\theta_{b}^{\prime}$.

(5) Go to step (2).

(6) Return $\theta_{c}, E_{\mathrm{D}}^{\mathrm{ZF}}\left(\theta_{c}\right)$, and $R_{\text {sum }}^{\infty}=R_{\text {sum }}^{\infty}\left(\theta_{c}, E_{\mathrm{D}}^{\mathrm{ZF}}\left(\theta_{c}\right)\right)$.

Algorithm 1: One-dimensional EB algorithm.

Similarly to the case of ZF processing, the above expression implies that the e2e performance with MRC/MRT processing can be improved by destination-aided EFs when it is limited by the $\mathrm{R} \rightarrow \mathrm{D}_{k}$ link.

Proposition 14. With MRC/MRT processing, the asymptotic sum rate is neither convex nor concave with respect to destination transmission power and the optimal destination transmission power that achieves the allowable maximum asymptotic sum rate is given by

$$
\begin{aligned}
& E_{D}^{M R C / M R T} \\
& = \begin{cases}0, & E_{S} \geq \frac{E_{M R C / M R T}}{\beta_{S R}} \\
\frac{E_{M R C / M R T}-E_{S} \beta_{S R}}{\beta_{D R}}, & E_{S}<\frac{E_{M R C / M R T}}{\beta_{S R}},\end{cases}
\end{aligned}
$$

where $E_{M R C / M R T} \triangleq\left(-b_{3}+c_{3}+\sqrt{\left(b_{3}-c_{3}\right)^{2}+4 a_{3} d_{3}}\right) / 2 a_{3}, a_{3} \triangleq$ $K / M E_{S} \sigma_{S R}^{2}, b_{3} \triangleq \sigma_{R}^{2} /(1-\theta) E_{S} \sigma_{S R}^{2}, c_{3} \triangleq K \beta_{D R} / M \sigma_{D R}^{2}$, and $d_{3} \triangleq \sigma_{D}^{2} / M \eta \theta \sigma_{D R}^{2}$.

Proof. By following similar procedure as the proof of Proposition 9 in Appendix E, Proposition 14 can be reached.

Proposition 15. With MRC/MRT processing, the asymptotic sum rate is concave with respect to $\theta$ and the optimal $\theta$ that achieves the allowable maximum asymptotic sum rate is given by

$$
\begin{aligned}
& \theta^{M R C / M R T} \\
& =\frac{a_{4}+b_{4}-c_{4}+d_{4}-\sqrt{\left(a_{4}+b_{4}-c_{4}\right)^{2}-2\left(a_{4}-b_{4}-c_{4}\right) d_{4}+d_{4}^{2}}}{2\left(a_{4}-c_{4}\right)},
\end{aligned}
$$

where $a_{4} \triangleq K\left(E_{S} \beta_{S R}+E_{D} \beta_{D R}\right) / M E_{S} \sigma_{S R}^{2}, b_{4} \triangleq \sigma_{R}^{2} / E_{S} \sigma_{S R}^{2}, c_{4} \triangleq$ $K \beta_{D R} / M \sigma_{D R}^{2}$, and $d_{4} \triangleq \sigma_{D}^{2} / M \eta \sigma_{D R}^{2}\left(E_{S} \beta_{S R}+E_{D} \beta_{D R}\right)$.

Proof. By following similar procedure as the proof of Proposition 10 in Appendix F, Proposition 15 can be reached.

Theorem 16. With MRC/MRT processing, the asymptotic sum rate is neither convex nor concave with respect to $\left(\theta, E_{D}\right)$ and there is a unique optimal $\left(\theta^{*}, E_{D}^{*}\right)$ that achieves the allowable maximum asymptotic sum rate.

Proof. By following similar procedure as the proof of Theorem 11 in Appendix G, Theorem 16 can be reached.

Since Propositions 14 and 15, and Theorem 16, have the similar expressions as those of Propositions 9 and 10, and Theorem 11, respectively, the one-dimensional EB algorithm can be applied to the case of MRC/MRT processing straightforwardly. Similarly to (40), the asymptotic harvested energy versus sum rate trade-off with MRC/MRT processing can be derived as

$$
\begin{aligned}
E_{\mathrm{h}}^{\infty}= & \tau_{\mathrm{u}} \eta K\left(\beta_{\mathrm{SR}} E_{\mathrm{S}}+\beta_{\mathrm{RD}} E_{\mathrm{D}}\right) \\
& \times \begin{cases}\frac{d_{4} \Lambda}{c_{4} \Lambda+1}, & \theta<\theta^{\mathrm{MRC} / \mathrm{MRT}} \\
1+\frac{b_{4} \Lambda}{a_{4} \Lambda-1}, & \theta \geq \theta^{\mathrm{MRC} / \mathrm{MRT}},\end{cases}
\end{aligned}
$$

where $a_{4}, b_{4}, c_{4}$, and $d_{4}$ are the same as those of Proposition 15 .

\section{Numerical Results}

This section presents some numerical results to verify the performance of the proposed protocol. For simplicity of illustration, the circuit power consumption and $\mathrm{EH}$ receiver sensitivity at the relay are ignored [21]. In all the illustrative examples, we choose $K=10, \tau_{\mathrm{p}}=2 K, \tau_{\mathrm{u}}=98, \eta=0.7, p_{\mathrm{p}}=$ $30 \mathrm{dBm}$, and $\sigma_{\mathrm{R}, \mathrm{p}}^{2}=\sigma_{\mathrm{R}}^{2}=\sigma_{\mathrm{D}}^{2}=\sigma^{2}=-90 \mathrm{dBm}$. Furthermore, we set $\mathbf{D}_{\mathrm{SR}}=\mathbf{D}_{\mathrm{DR}}=\beta \mathbf{I}_{K}$ to model i.i.d. fading, where $\beta=-65 \mathrm{~dB}$ corresponds to a path-loss related to a distance of about $10 \mathrm{~m}$ for the carrier frequency of $915 \mathrm{MHz}$.

In Figure 2, the trade-off between the harvested energy and achievable sum rate is investigated for $\mathrm{ZF}$ processing. Five levels of destination-aided EFs, that is, $E_{\mathrm{D}}=35 \mathrm{dBm}$, $E_{\mathrm{D}}=30 \mathrm{dBm}, E_{\mathrm{D}}=20 \mathrm{dBm}, E_{\mathrm{D}}=0 \mathrm{~mW}$, and $E_{\mathrm{D}}^{\mathrm{ZF}}$, are considered, respectively, whereas $E_{\mathrm{S}}$ is fixed at $25 \mathrm{dBm}$. Note that $E_{\mathrm{D}}=0 \mathrm{~mW}$ corresponds to the scenario where the destinations do not transmit. The asymptotic trade-off curves are plotted by using (40). Furthermore, a set of trade-off curves are plotted by using Monte Carlo simulations with 


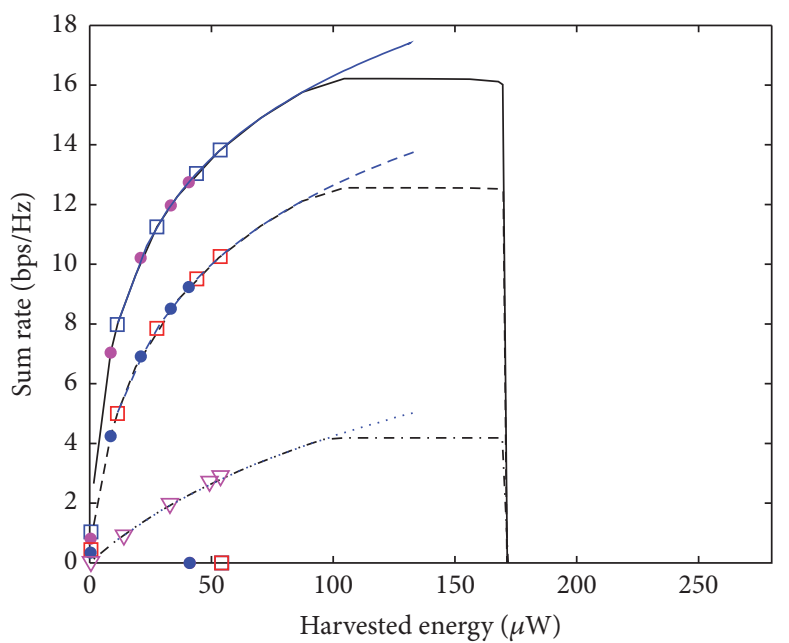

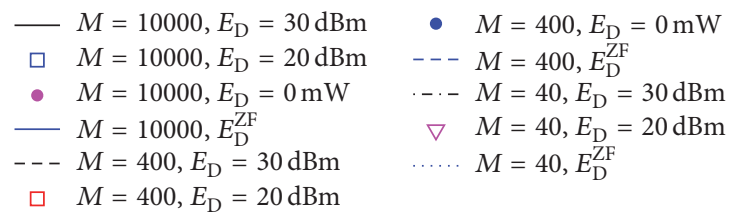

FIgURE 2: Trade-off between $E_{\mathrm{h}}$ and sum rate with ZF processing.

gradually increasing $M$ from 40 to 10000. In Figure 2, the points of the trade-off curves corresponding to a higher harvested energy refer to a larger PS ratios and, consequently, this scenario leads to a higher sum rate. Nevertheless, as $\theta \rightarrow$ 1 , the achievable sum rates of all the fixed $E_{\mathrm{D}}$ drop down dramatically and approach zero. It can be seen from Figure 2 that the trade-off between the harvested energy and sum rate can be improved significantly by using a very large antenna array. For example, for $E_{\mathrm{D}}$ equal to $20 \mathrm{dBm}$ and $30 \mathrm{dBm}$, destination-aided EFs can effectively improve the trade-off between harvested energy and sum rate. However, for $E_{\mathrm{D}}=$ $35 \mathrm{dBm}$, destination-aided EFs deteriorate the trade-off. Furthermore, since a fixed $E_{\mathrm{D}}$ can only achieve a part of the sum rate compared to that of $E_{\mathrm{D}}^{\mathrm{ZF}}$, Figure 2 shows that the curves are overlapped partially, whereas $E_{\mathrm{D}}^{\mathrm{ZF}}$ achieves the highest trade-off between harvested energy and sum rate.

In Figure 3, we investigate the trade-off between the harvested energy and achievable sum rate for MRC/MRT processing. Four levels of destination-aided EFs, that is, $E_{\mathrm{D}}=$ $30 \mathrm{dBm}, E_{\mathrm{D}}=20 \mathrm{dBm}, E_{\mathrm{D}}=0 \mathrm{~mW}$, and $E_{\mathrm{D}}^{\mathrm{ZF}}$, are considered, respectively, whereas $E_{\mathrm{S}}$ is fixed at $25 \mathrm{dBm}$. The asymptotic trade-off curves are plotted by using (46). The trade-off curves for $M \rightarrow \infty$ are also presented in Figure 3. As can be seen from Figure 3, the trade-off curves for $M \rightarrow \infty$ serve as the trade-off bounds. When $M$ is a limited number, similar to the case of ZF processing, a larger PS ratio leads to a higher harvested energy as well as a higher achievable sum rate. Also, the achievable sum rate drops down dramatically as $\theta \rightarrow 1$.

In Figures 4 and 5, we investigate sum rate versus $E_{\mathrm{D}}$ for ZF processing and MRC/MRT processing, respectively. The curves in Figures 4 and 5 verify the correctness of Propositions $9,10,14$, and 15. Compared with fixed $\theta$ and fixed $E_{\mathrm{D}}$,
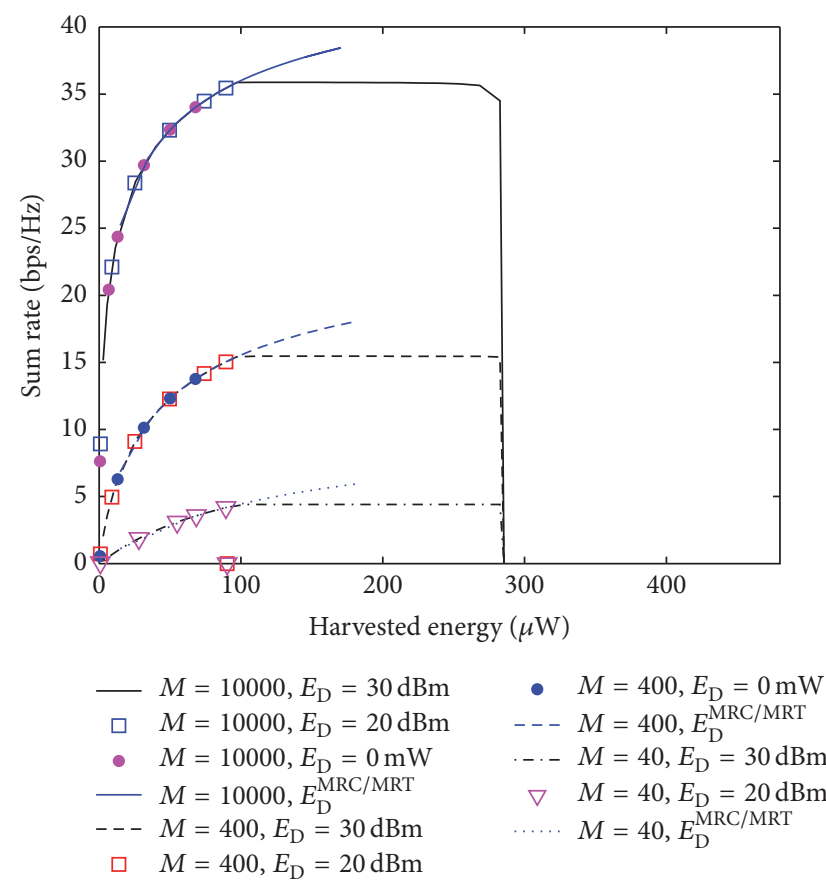

FIgURE 3: Trade-off between $E_{\mathrm{h}}$ and sum rate with MRC/MRT processing.

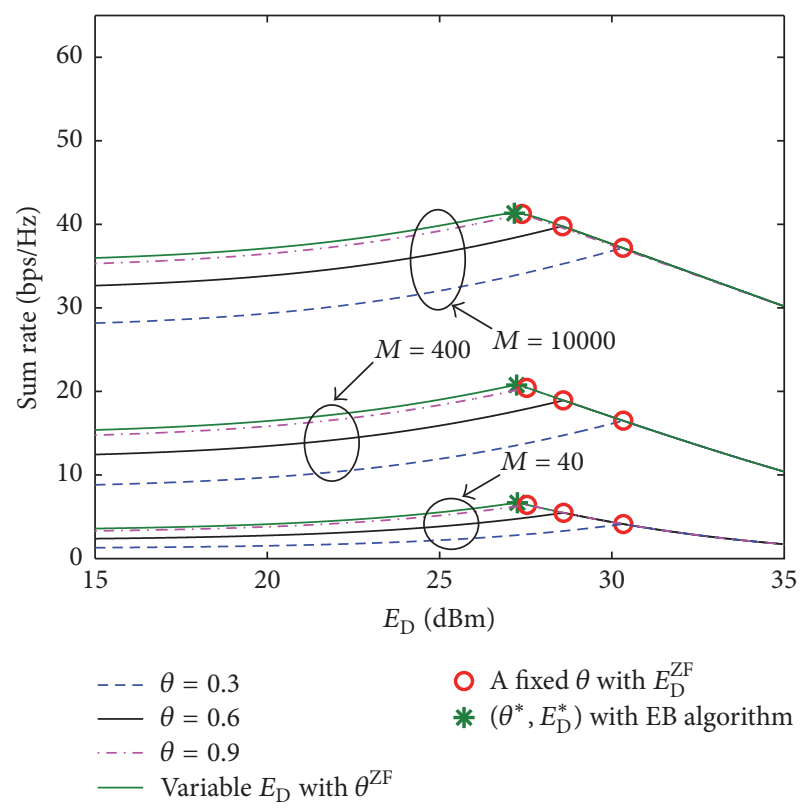

FIGURE 4: Sum rate versus $E_{\mathrm{D}}$ with $\mathrm{ZF}$ processing.

the corresponding $E_{\mathrm{D}}^{\mathrm{ZF}}\left(E_{\mathrm{D}}^{\mathrm{MRC} / \mathrm{MRT}}\right)$ and $\theta^{\mathrm{ZF}}\left(\theta^{\mathrm{MRC} / \mathrm{MRT}}\right)$ can, respectively, improve the sum rate. Furthermore, Figures 4 and 5 show that the proposed one-dimensional $\mathrm{EB}$ algorithm achieves the allowable maximum sum rate. Figures 4 and 5 also show that the sum rate increases as $M$ increases from 40 to 10000 .

In Figures 6 and 7, we investigate sum rate versus PS factor for ZF processing and MRC/MRT processing, respectively. 


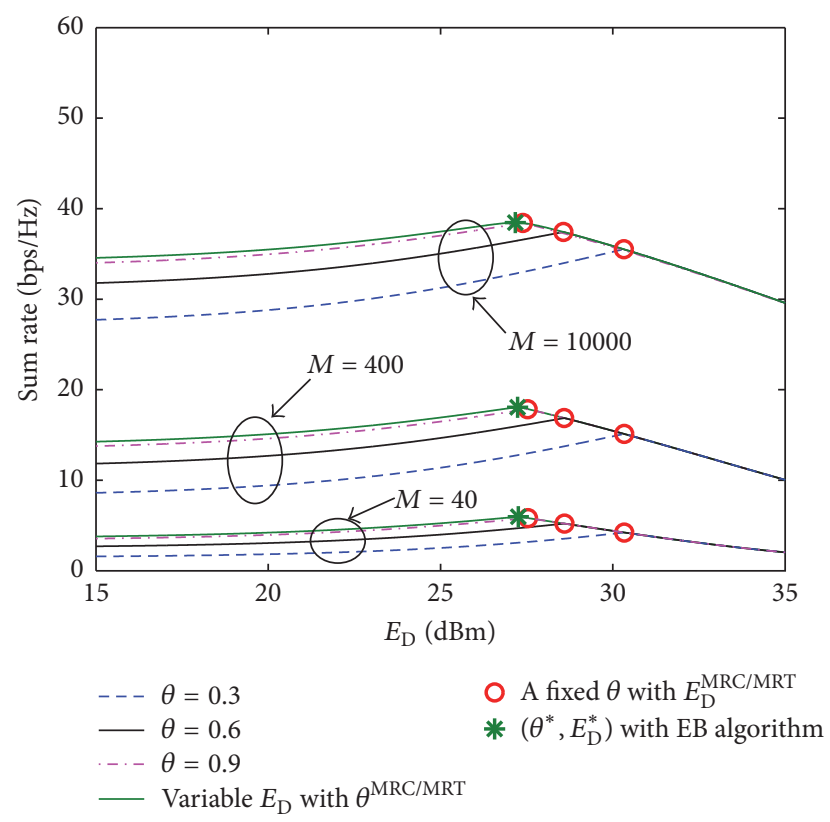

Figure 5: Sum rate versus $E_{\mathrm{D}}$ with MRC/MRT processing.

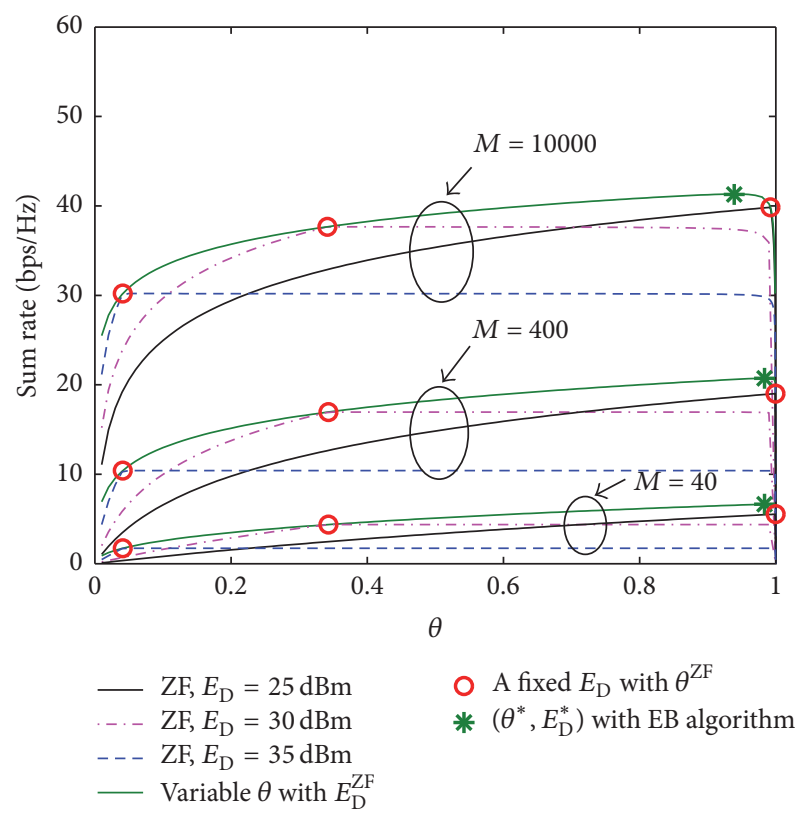

FIgURE 6: Sum rate versus $\theta$ with ZF processing.

The results in Figures 6 and 7 verify the correctness of the analytical $\theta^{\mathrm{ZF}}$ and $\theta^{\mathrm{MRC} / \mathrm{MRT}}$, respectively. As can be seen from Figures 6 and 7, the optimal PS factor almost keeps unchanged with the variable $M$ for the considered same power budget. Furthermore, the optimal PS factor for ZF processing is almost the same as that for MRC/MRT processing. Moreover, Figures 6 and 7 verify that the sum rate is concave with respect to $\theta$. Figures 6 and 7 also verify that the onedimensional EB algorithm achieves the allowable maximum sum rate.

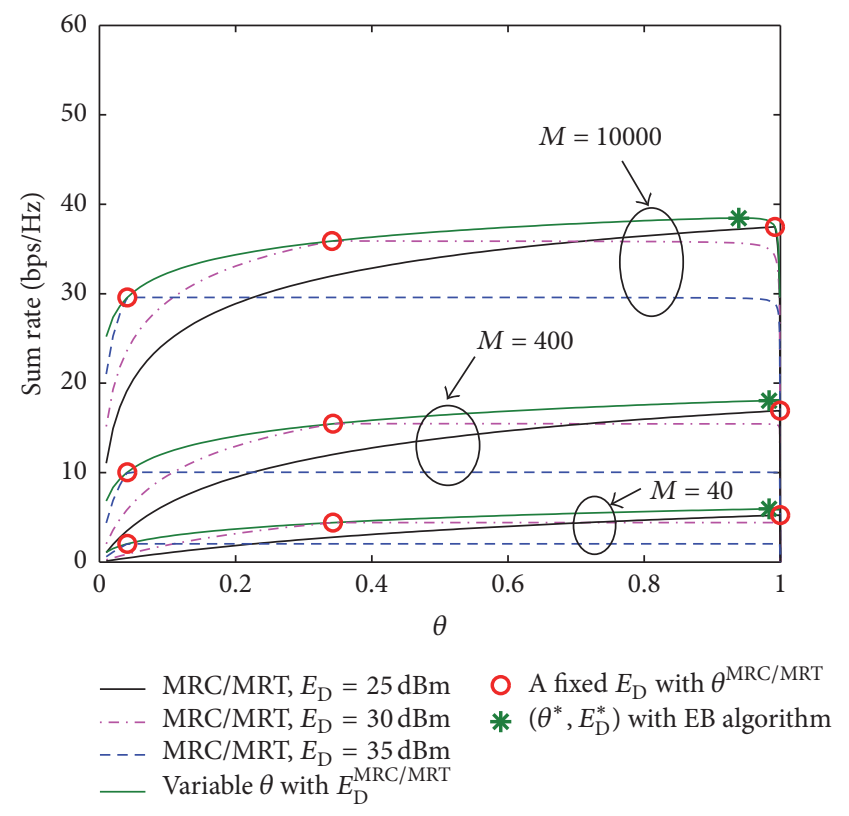

Figure 7: Sum rate versus $\theta$ with MRC/MRT processing.

In Figures 8 and 9, average sum rates are plotted against source transmission power for ZF processing and MRC/MRT processing, respectively. The analytical curves are plotted by using (34) and (42) for ZF processing and MRC/MRT processing, respectively, whereas the exact sum rate curves are plotted by using Monte Carlo simulations. Figures 8 and 9 clearly show that the closed-form achievable rates in (34) and (42) are accurate in computing the sum rate based on channel statistics-aided decoding with a finite number of relay antennas. Furthermore, it can be clearly seen from Figures 8 and 9 that destination-aided EFs can improve the sum rate significantly. This verifies that ELI can be effectively cancelled out by massive MIMO processing. Moreover, the highest sum rate is achieved by the proposed one-dimensional EB algorithm. Note that $E_{\mathrm{D}}^{\mathrm{ZF}}$ and $E_{\mathrm{D}}^{\mathrm{ZF}}$ achieve the second highest sum rate in Figures 8 and 9, respectively, the correctness of Propositions 9 and 14 is verified, respectively. Also, the piecewise sum rate curves verify the correctness of Propositions 10 and 15 . By comparing the curves of $M=40$ and $M=10000$, we observe that the gains of sum rate can be obtained by increasing the number of relay antennas while keeping the source transmission power in useful regions. Notably, Figures 8 and 9 show that the sum rate achieved by ZF processing is higher than that of MRC/MRT processing in the high $E_{S}$ region, whereas both ZF processing and MRC/MRT processing achieve almost the same sum rate in the low $E_{\mathrm{S}}$ region.

The sum rate versus the number of the relay antennas is investigated in Figure 10 with $E_{\mathrm{S}}=25 \mathrm{dBm}$. As $M$ increases, the corresponding sum rate increases accordingly. The curves in Figure 10 show that ZF processing achieves a higher sum rate than that of MRC/MRT processing with the considered power budget. Furthermore, in the whole region of the value of $M$, the highest sum rate is achieved by the one-dimensional $\mathrm{EB}$ algorithm and the second highest sum rate is achieved by 


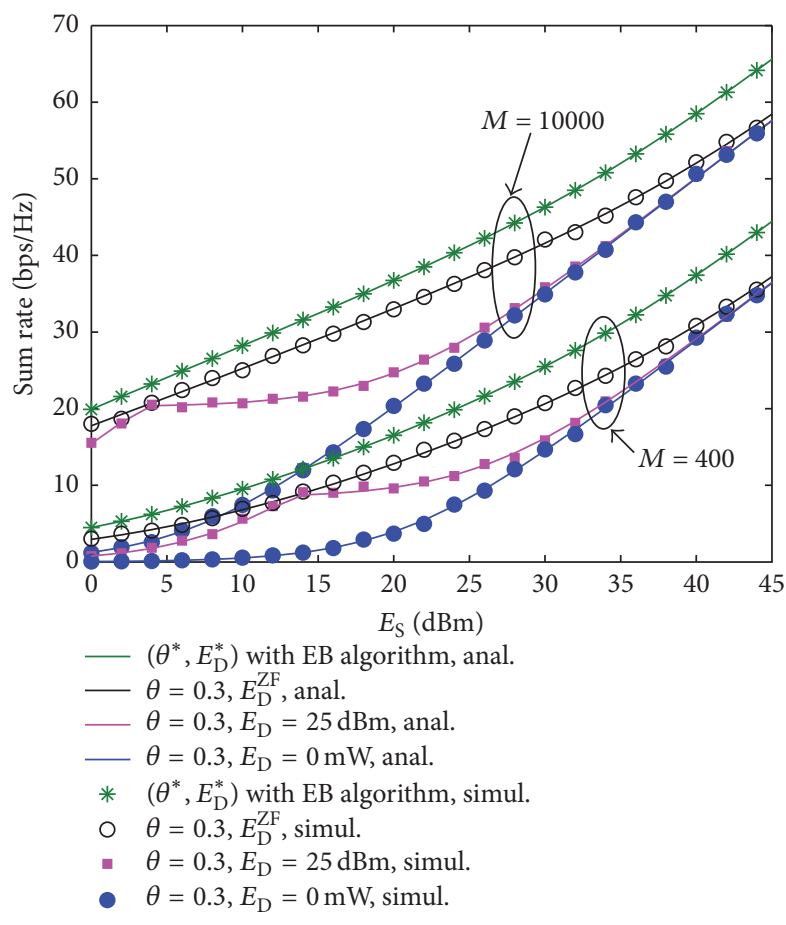

FIgURE 8: Sum rate versus $E_{\mathrm{S}}$ with $\mathrm{ZF}$ processing.
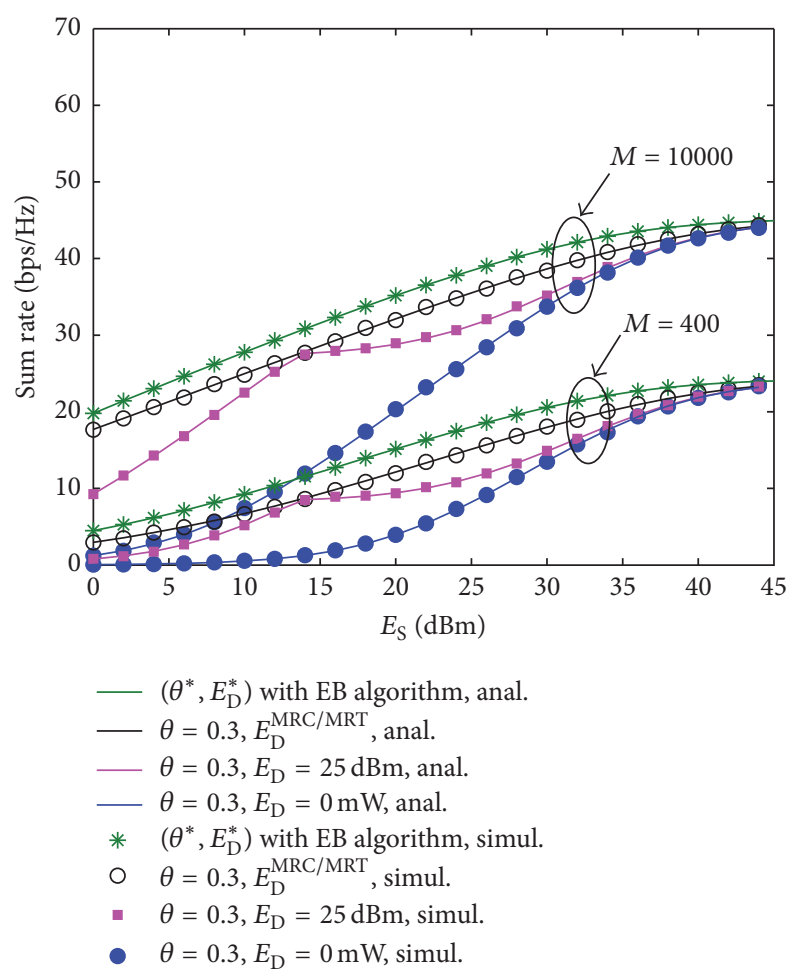

FIGURE 9: Sum rate versus $E_{\mathrm{S}}$ with MRC/MRT processing.

$E_{\mathrm{D}}^{\mathrm{ZF}}$ (and $E_{\mathrm{D}}^{\mathrm{MRC} / \mathrm{MRT}}$ ). Compared to $E_{\mathrm{D}}=0 \mathrm{~mW}$, the proposed destination-aided EFs can significantly improve the sum rate in the whole region of the value of $M$.

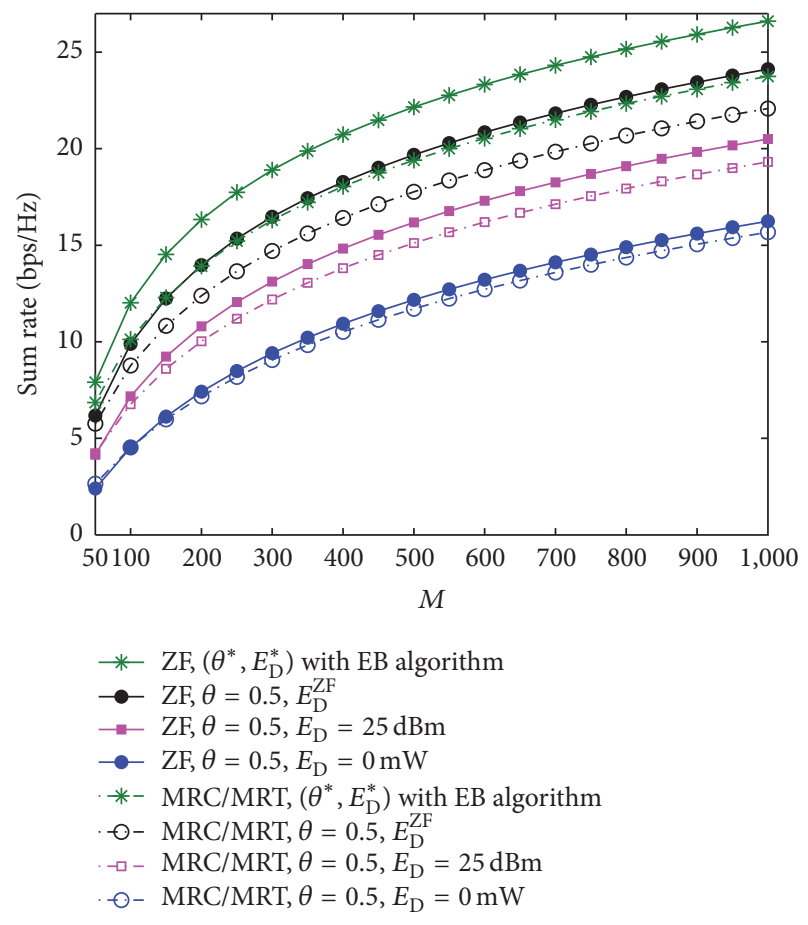

FIGURE 10: Sum rate versus the number of the relay antennas.

\section{Conclusion}

A destination-aided SWIPT relaying protocol has been proposed for a multipair massive MIMO relay network, in which a PS relay employs linear processing to cancel MI and ELI. The expressions of asymptotic harvested energy and symmetric sum rate with massive MIMO relay have been derived in closed-form. The trade-off between asymptotic harvested energy and achievable sum rate has been quantified. The effect of destination-aided EFs on the sum rate has been investigated and our results reveal that destinationaided EFs can boost the energy harvesting, so that achievable sum rate can be significantly improved by destination-aided EFs. Meanwhile, the detrimental impact of ELI on sum rate performance can be cancelled out by using a massive MIMO relay with linear processing. It has shown that asymptotic sum rate is neither convex nor concave with respect to PS and destination transmission power and a one-dimensional EB algorithm has been proposed to obtain the optimal PS and destination transmission power. The significant sum rate improvement of the proposed scheme has been verified by numerical results.

\section{Appendix}

\section{A. Proof of Proposition 1}

First, we recall two important identities for random matrices. As $M \rightarrow \infty$, the column vectors of $\mathbf{H}_{\mathrm{SR}}$ and $\mathbf{H}_{\mathrm{DR}}$ become orthogonal to each other; that is,

$$
\frac{\mathbf{h}_{\mathrm{SR}, i}^{H} \mathbf{h}_{\mathrm{SR}, j}}{M} \stackrel{\text { a.s. }}{\longrightarrow} \begin{cases}1, & i=j \\ 0, & i \neq j,\end{cases}
$$




$$
\frac{\mathbf{h}_{\mathrm{DR}, i}^{H} \mathbf{h}_{\mathrm{DR}, j}}{M} \stackrel{\text { a.s. }}{\longrightarrow} \begin{cases}1, & i=j \\ 0, & i \neq j,\end{cases}
$$

where $\mathbf{h}_{\mathrm{SR}, k}$ and $\mathbf{h}_{\mathrm{DR}, k}$ are the $k$ th columns of $\mathbf{H}_{\mathrm{SR}}$ and $\mathbf{H}_{\mathrm{SR}}$, respectively. Consequently, as $M \rightarrow \infty$, it can be shown that

$$
\begin{gathered}
\frac{\mathbf{G}_{\mathrm{SR}}^{H} \mathbf{G}_{\mathrm{SR}}}{M} \stackrel{\text { a.s. }}{\longrightarrow} \mathbf{D}_{\mathrm{SR}}, \\
\frac{\mathbf{G}_{\mathrm{DR}}^{H} \mathbf{G}_{\mathrm{DR}}}{M} \stackrel{\text { a.s. }}{\longrightarrow} \mathbf{D}_{\mathrm{DR}} .
\end{gathered}
$$

By substituting $P_{\mathrm{S}}=E_{\mathrm{S}} / M$ and $P_{\mathrm{D}}=E_{\mathrm{D}} / M$ into (4), the harvested energy at the $\mathrm{EH}$ receiver of $\mathrm{R}$ can be rewritten as

$$
\begin{aligned}
& E_{\mathrm{h}} \\
& =\tau_{\mathrm{u}} \eta \theta\left(E_{\mathrm{S}} \operatorname{Tr}\left(\frac{\mathbf{G}_{\mathrm{SR}}^{H} \mathbf{G}_{\mathrm{SR}}}{M}\right)+E_{\mathrm{D}} \operatorname{Tr}\left(\frac{\mathbf{G}_{\mathrm{DR}}^{H} \mathbf{G}_{\mathrm{DR}}}{M}\right)\right) .
\end{aligned}
$$

Based on the fact of (A.2), whenever the number of antennas at the relay grows without bound, the harvested energy expression in (A.3) can be derived as

$$
E_{\mathrm{h}} \stackrel{\text { a.s. }}{\longrightarrow} \tau_{\mathrm{u}} \eta \theta\left(E_{\mathrm{S}} \operatorname{Tr}\left(\mathbf{D}_{\mathrm{SR}}\right)+E_{\mathrm{D}} \operatorname{Tr}\left(\mathbf{D}_{\mathrm{DR}}\right)\right) .
$$

\section{B. Proof of Proposition 3}

(1) For ZF Processing. By substituting (9) and (14) into (10), the processed signal at the relay before amplification can be expressed as

$$
\begin{aligned}
& \sqrt{(1-\theta) P_{\mathrm{S}}} \mathbf{W}^{H} \mathbf{G}_{\mathrm{SR}} \mathbf{x}_{\mathrm{S}} \\
& =\sqrt{(1-\theta) P_{\mathrm{S}}} \mathbf{W}^{H}\left(\widehat{\mathbf{G}}_{\mathrm{SR}}+\mathscr{E}_{\mathrm{SR}}\right) \mathbf{x}_{\mathrm{S}} \\
& =\sqrt{(1-\theta) P_{\mathrm{S}}} \mathbf{x}_{\mathrm{S}}+\sqrt{(1-\theta) P_{\mathrm{S}}} \mathbf{W}^{H} \mathscr{E}_{\mathrm{SR}} \mathbf{x}_{\mathrm{S}} .
\end{aligned}
$$

By using the law of large numbers, we have

$$
\begin{aligned}
& \sqrt{(1-\theta) P_{\mathrm{S}}} \mathbf{W}^{H} \mathscr{E}_{\mathrm{SR}} \mathbf{x}_{\mathrm{S}} \\
& =\sqrt{(1-\theta) p_{s}}\left(\frac{\widehat{\mathbf{G}}_{\mathrm{SR}}^{H} \widehat{\mathbf{G}}_{\mathrm{SR}}}{M}\right)^{-1} \frac{\widehat{\mathbf{G}}_{\mathrm{SR}}^{H} \mathscr{E}_{\mathrm{SR}}}{M} \mathbf{x}_{\mathrm{S}} \stackrel{\text { a.s. }}{\longrightarrow} \mathbf{0}, \\
& \text { as } M \rightarrow \infty .
\end{aligned}
$$

Therefore, as $M \rightarrow \infty$, the received and processed signal at the relay for detection satisfies

$$
\sqrt{(1-\theta) P_{\mathrm{S}}} \mathbf{W}^{H} \mathbf{G}_{\mathrm{SR}} \mathbf{x}_{\mathrm{S}} \stackrel{\text { a.s. }}{\longrightarrow} \sqrt{(1-\theta) P_{\mathrm{S}}} \mathbf{x}_{\mathrm{S}} .
$$

The above expression can be rewritten in the element form as

$$
\begin{aligned}
& \sqrt{(1-\theta) P_{\mathrm{S}}} \mathbf{w}_{k}^{H} \mathbf{g}_{\mathrm{SR}, k} x_{\mathrm{S}, k} \stackrel{\text { a.s. }}{\longrightarrow} \sqrt{(1-\theta) P_{\mathrm{S}}} x_{\mathrm{S}, k}, \\
& \sqrt{(1-\theta) P_{\mathrm{S}}} \mathbf{w}_{k}^{H} \mathbf{g}_{\mathrm{SR}, j} x_{\mathrm{S}, j} \stackrel{\text { a.s. }}{\longrightarrow} 0
\end{aligned}
$$

Next, we consider the ELI term. Note that the vector $\widehat{\mathbf{g}}_{\mathrm{SR}, k}$ is independent of the vector $\widehat{\mathbf{g}}_{\mathrm{DR}, k}$. With ZF processing, it can be shown by using the law of large numbers that

$$
\begin{aligned}
& \sqrt{(1-\theta) P_{\mathrm{D}}} \mathbf{W}^{H} \mathbf{G}_{\mathrm{DR}} \mathbf{x}_{\mathrm{D}} \\
& =\alpha_{\mathrm{ZF}} \sqrt{(1-\theta) P_{\mathrm{D}}}\left(\frac{\widehat{\mathbf{G}}_{\mathrm{SR}}^{H} \widehat{\mathbf{G}}_{\mathrm{SR}}}{M}\right)^{-1} \frac{\widehat{\mathbf{G}}_{\mathrm{SR}}^{H} \mathbf{G}_{\mathrm{DR}}}{M} \mathbf{x}_{\mathrm{D}} \\
& \stackrel{\text { a.s. }}{\longrightarrow} \mathbf{0}, \quad \text { as } M \longrightarrow \infty .
\end{aligned}
$$

Similarly, it can be shown that

$$
\mathbf{W}^{H} \mathbf{n}_{\mathrm{R}} \stackrel{\text { a.s. }}{\longrightarrow} \mathbf{0} \text {. }
$$

By substituting (B.4), the element form of (B.5), and the element form of (B.6) into (11), we arrive at (21).

(2) For MRC/MRT Processing. By using the law of large numbers, the desired signal in (11) converges to a deterministic value when the receive antenna count goes to infinity; that is, as $M \rightarrow \infty$,

$$
\frac{\sqrt{(1-\theta) P_{\mathrm{S}}}}{M} \widehat{\mathbf{g}}_{\mathrm{SR}, k}^{H} \mathbf{g}_{\mathrm{SR}, k} x_{\mathrm{S}, k} \stackrel{\text { a.s. }}{\longrightarrow} \sqrt{(1-\theta) P_{\mathrm{S}}} \sigma_{\mathrm{SR}, k}^{2} x_{\mathrm{S}, k} .
$$

For the $\mathrm{MI}$, as $M \rightarrow \infty$,

$$
\frac{\sqrt{(1-\theta) P_{\mathrm{S}}}}{M} \widehat{\mathbf{g}}_{\mathrm{SR}, k}^{H} \mathbf{g}_{\mathrm{SR}, j} x_{\mathrm{S}, j} \stackrel{\text { a.s. }}{\longrightarrow} 0, \quad \forall j \neq k .
$$

We next consider the ELI, $\sqrt{(1-\theta) P_{\mathrm{D}}} \widehat{\mathbf{g}}_{\mathrm{SR}, k}^{H} \mathbf{G}_{\mathrm{DR}} \mathbf{x}_{\mathrm{D}}$. As $M \rightarrow$ $\infty$, by using the law of large number, the $k$ th element of the $1 \times$ $K$ row vector, $\left(\sqrt{(1-\theta) P_{\mathrm{D}}} / M\right) \widehat{\mathbf{g}}_{\mathrm{SR}, k}^{H} \mathbf{G}_{\mathrm{DR}}$, can be shown to be $\left(\sqrt{(1-\theta) P_{\mathrm{D}}} / M\right) \widehat{\mathbf{g}}_{\mathrm{SR}, k}^{\mathrm{H}} \mathbf{g}_{\mathrm{DR}, k} \stackrel{\text { a.s. }}{\longrightarrow} 0$ due to the fact that $\widehat{\mathbf{g}}_{\mathrm{SR}, k}$ and $\mathbf{g}_{\mathrm{DR}, k}$ are statistically independent. Thus, the ELI converges to 0 as $M \rightarrow \infty$; that is,

$$
\frac{\sqrt{(1-\theta) P_{\mathrm{D}}}}{M} \widehat{\mathbf{g}}_{\mathrm{SR}, k}^{H} \mathbf{G}_{\mathrm{DR}} \mathbf{x}_{\mathrm{D}} \stackrel{\text { a.s. }}{\longrightarrow} 0 .
$$

Since $\mathbf{g}_{\mathrm{SR}, k}$ and $\mathbf{n}_{\mathrm{R}}$ are also statistically independent, as $M \rightarrow$ $\infty$, it can be shown that

$$
\frac{1}{M} \widehat{\mathbf{g}}_{\mathrm{SR}, k}^{H} \mathbf{n}_{\mathrm{R}} \stackrel{\text { a.s. }}{\longrightarrow} 0 .
$$

Substituting (B.7), (B.8), (B.9), and (B.10) into (11), we obtain (22).

\section{Proof of Proposition 5}

(1) For ZF Processing. By using [25, Lemma 2.10] and the law of large numbers, as $M \rightarrow \infty$, we have

$$
\alpha_{\mathrm{ZF}} \stackrel{\text { a.s. }}{\longrightarrow} \sqrt{\frac{M-K}{\operatorname{Tr}\left(\mathbf{D}_{\mathrm{RD}}^{-1}\right)}} .
$$


Substituting (C.1) into (15), it can be shown that

$$
\begin{aligned}
& \mathbf{g}_{\mathrm{DR}, k}^{T} \mathbf{a}_{k} x_{\mathrm{S}, k} \\
& \stackrel{\text { a.s. }}{\longrightarrow} M \sqrt{\frac{M-K}{\operatorname{Tr}\left(\widehat{\mathbf{D}}_{\mathrm{RD}}^{-1}\right)}} \operatorname{Tr}\left(\frac{\left(\widehat{\mathbf{G}}_{\mathrm{RD}}^{H} \widehat{\mathbf{G}}_{\mathrm{RD}}\right)^{-1}}{M}\right) \sigma_{\mathrm{DR}, k}^{2} x_{\mathrm{S}, k} \\
& \quad=M \sqrt{(M-K) \operatorname{Tr}\left(\widehat{\mathbf{D}}_{\mathrm{RD}}^{-1}\right)} \sigma_{\mathrm{DR}, k}^{2} x_{\mathrm{S}, k} .
\end{aligned}
$$

Furthermore, by substituting (20) into $P_{\mathrm{R}}=E_{\mathrm{h}} / T$, as $M \rightarrow$ $\infty$, we have

$$
P_{\mathrm{R}} \stackrel{\text { a.s. }}{\longrightarrow} \eta \theta\left(E_{\mathrm{S}} \operatorname{Tr}\left(\mathbf{D}_{\mathrm{SR}}\right)+E_{\mathrm{D}} \operatorname{Tr}\left(\mathbf{D}_{\mathrm{DR}}\right)\right) .
$$

Thus, by substituting (C.2) and (C.3) into the term $\sqrt{P_{\mathrm{R}}} \mathbf{g}_{\mathrm{DR}, k}^{T} \mathbf{a}_{k} x_{\mathrm{S}, k}$, as $M \rightarrow \infty$, we obtain

$$
\begin{aligned}
& \frac{\sqrt{P_{\mathrm{R}}} \mathbf{g}_{\mathrm{DR}, k}^{T} \mathbf{a}_{k} x_{\mathrm{S}, k}}{M \sqrt{M-K}} \\
& \stackrel{\text { a.s. }}{\longrightarrow} \sqrt{\eta \theta \sigma_{\mathrm{DR}, k}^{4} \operatorname{Tr}\left(\mathbf{D}_{\mathrm{RD}}^{-1}\right)\left(E_{\mathrm{S}} \operatorname{Tr}\left(\mathbf{D}_{\mathrm{SR}}\right)+E_{\mathrm{D}} \operatorname{Tr}\left(\mathbf{D}_{\mathrm{DR}}\right)\right)} x_{\mathrm{S}, k} .
\end{aligned}
$$

Moreover, as $M \rightarrow \infty$, it can be shown that

$$
\begin{gathered}
\frac{\sqrt{P_{\mathrm{R}}}}{M \sqrt{M-K}} \mathbf{g}_{\mathrm{DR}, k}^{T} \mathbf{a}_{j}^{*} x_{\mathrm{S}, j} \stackrel{\text { a.s. }}{\longrightarrow} 0, \quad \forall j \neq k, \\
\frac{1}{M \sqrt{M-K}} \mathbf{n}_{\mathrm{D}, k} \stackrel{\text { a.s. }}{\longrightarrow} 0 .
\end{gathered}
$$

By substituting (C.4) and (C.5) into (13), we obtain (23).

(2) For MRC/MRT Processing. With MRC/MRT processing, by using the law of large numbers, we have

$$
\frac{1}{\sqrt{M}} \mathbf{g}_{\mathrm{DR}, k}^{T} \widehat{\mathbf{g}}_{\mathrm{DR}, k}^{*} x_{\mathrm{S}, k} \stackrel{\text { a.s. }}{\longrightarrow} \sigma_{\mathrm{DR}, k}^{2} x_{\mathrm{S}, k} .
$$

The normalization factor can be rewritten as

$$
\alpha=\frac{1}{\sqrt{M \operatorname{Tr}\left(\widehat{\mathbf{G}}_{\mathrm{RD}}^{H} \widehat{\mathbf{G}}_{\mathrm{RD}} / M\right)}} .
$$

As $M \rightarrow \infty$, it can be shown that $\alpha \sqrt{M} \stackrel{\text { a.s. }}{\longrightarrow} 1 / \sqrt{\operatorname{Tr}\left(\widehat{\mathbf{D}}_{\mathrm{DR}}\right)}$. Thus, by substituting (C.6), (C.7), and (C.3) into the term $\alpha \sqrt{P_{\mathrm{R}}} \mathbf{g}_{\mathrm{DR}, k}^{T} \mathbf{g}_{\mathrm{DR}, k}^{*} x_{\mathrm{S}, k}$, as $M \rightarrow \infty$, we obtain

$$
\begin{aligned}
& \frac{\alpha \sqrt{P_{\mathrm{R}}} \mathbf{g}_{\mathrm{DR}, k}^{T} \widehat{\mathbf{g}}_{\mathrm{DR}, k}^{*} x_{\mathrm{S}, k}}{\sqrt{M}} \\
& \stackrel{\text { a.s. }}{\longrightarrow} \sqrt{\frac{\eta \theta \sigma_{\mathrm{DR}, k}^{4}\left(E_{\mathrm{S}} \operatorname{Tr}\left(\mathbf{D}_{\mathrm{SR}}\right)+E_{\mathrm{D}} \operatorname{Tr}\left(\mathbf{D}_{\mathrm{DR}}\right)\right)}{\operatorname{Tr}\left(\widehat{\mathbf{D}}_{\mathrm{DR}}\right)}} x_{\mathrm{S}, k} .
\end{aligned}
$$

Moreover, as $M \rightarrow \infty$, it can be shown that

$$
\begin{gathered}
\frac{\alpha \sqrt{P_{\mathrm{R}}}}{\sqrt{M}} \mathbf{g}_{\mathrm{DR}, k}^{T} \widehat{\mathbf{g}}_{\mathrm{DR}, j}^{*} x_{\mathrm{S}, j} \stackrel{\text { a.s. }}{\longrightarrow} 0, \quad \forall j \neq k, \\
\frac{1}{\sqrt{M}} \mathbf{n}_{\mathrm{D}, k} \stackrel{\text { a.s. }}{\longrightarrow} 0 .
\end{gathered}
$$

By substituting (C.8) and (C.9) into (13), we obtain (24).

\section{Proof of Theorem 7}

(1) Derive $R_{S R, k}$. To derive analytical expression for (29), we first compute $\mathbb{E}\left\{\mathbf{w}_{k}^{H} \mathbf{g}_{\mathrm{SR}, k}\right\}, \operatorname{Var}\left(\mathbf{w}_{k}^{H} \mathbf{g}_{\mathrm{SR}, k}\right), \mathrm{MI}_{k}, \mathrm{ELI}_{k}$, and $\mathrm{AN}_{k}$, respectively.

(i) Compute $\mathbb{E}\left\{\mathbf{w}_{k}^{H} \mathbf{g}_{S R, k}\right\}$. Substituting (14) and (9) into $\mathbf{W}^{H} \mathbf{G}_{\mathrm{SR}}$, we have

$$
\mathbf{W}^{H} \mathbf{G}_{\mathrm{SR}}=\mathbf{W}^{H}\left(\widehat{\mathbf{G}}_{\mathrm{SR}}+\mathscr{E}_{\mathrm{SR}}\right)=\mathbf{I}_{M}+\mathbf{W}^{H} \mathscr{E}_{\mathrm{SR}}
$$

which shows that

$$
\mathbf{w}_{k}^{H} \mathbf{g}_{\mathrm{SR}, k}=1+\mathbf{w}_{k}^{H} \mathscr{E}_{\mathrm{SR}, k},
$$

where $\mathscr{E}_{\mathrm{SR}, k}$ is the $k$ th column of $\mathscr{E}_{\mathrm{SR}}$. Since the zero-meanvalued $\mathscr{E}_{\mathrm{SR}, k}$ is independent of $\mathbf{w}_{k}$, we have $\mathbb{E}\left\{\mathbf{w}_{k}^{H} \mathscr{E}_{\mathrm{SR}, k}\right\}=0$. Thus,

$$
\mathbb{E}\left\{\mathbf{w}_{k}^{H} \mathbf{g}_{\mathrm{SR}, k}\right\}=1 \text {. }
$$

(ii) Compute $\operatorname{Var}\left(\mathbf{w}_{k}^{H} \mathbf{g}_{S R, k}\right)$. From (D.2) and (D.3), the variance of $\mathbf{w}_{k}^{H} \mathbf{g}_{\mathrm{SR}, k}$ can be expressed as

$$
\begin{aligned}
\operatorname{Var} & \left(\mathbf{w}_{k}^{H} \mathbf{g}_{\mathrm{SR}, k}\right)=\mathbb{E}\left\{\left|\mathbf{w}_{k}^{H} \mathscr{E}_{\mathrm{SR}, k}\right|^{2}\right\} \\
= & \left(\beta_{\mathrm{SR}, k}-\sigma_{\mathrm{SR}, k}^{2}\right) \mathbb{E}\left\{\left\|\mathbf{w}_{k}\right\|^{2}\right\} \\
= & \left(\beta_{\mathrm{SR}, k}-\sigma_{\mathrm{SR}, k}^{2}\right) \mathbb{E}\left\{\left[\left(\widehat{\mathbf{G}}_{\mathrm{SR}}^{H} \widehat{\mathbf{G}}_{\mathrm{SR}}\right)^{-1}\right]_{k k}\right\} \\
= & \frac{\beta_{\mathrm{SR}, k}-\sigma_{\mathrm{SR}, k}^{2}}{K \sigma_{\mathrm{SR}, k}^{2}} \mathbb{E}\left\{\operatorname{Tr}\left(\mathbf{X}^{-1}\right)\right\}=\frac{\beta_{\mathrm{SR}, k}-\sigma_{\mathrm{SR}, k}^{2}}{(M-K) \sigma_{\mathrm{SR}, k}^{2}},
\end{aligned}
$$

where $\mathbf{X} \triangleq \widehat{\mathbf{G}}_{\mathrm{SR}}^{\mathbf{H}} \widehat{\mathbf{G}}_{\mathrm{SR}}$ is a $K \times K$ central Wishart matrix with $M$ degrees of freedom and covariance matrix $\mathbf{I}_{K},[\cdot]_{k k}$ denotes the $(k, k)$ th element of a matrix, and the last equality is obtained by using [25, Lemma 2.10].

(iii) Compute $M I_{k}$. Since $\mathbf{w}_{k}^{H} \mathbf{g}_{\mathrm{SR}, j}=\mathbf{w}_{k}^{H} \mathscr{E}_{\mathrm{SR}, j}$, for $j \neq k$, we have

$$
\begin{aligned}
\mathbb{E}\left\{\left|\mathbf{w}_{k}^{H} \mathbf{g}_{\mathrm{SR}, j}\right|^{2}\right\} & =\mathbb{E}\left\{\left|\mathbf{w}_{k}^{H} \mathscr{E}_{\mathrm{SR}, j}\right|^{2}\right\} \\
& =\left(\beta_{\mathrm{SR}, j}-\sigma_{\mathrm{SR}, j}^{2}\right) \mathbb{E}\left\{\left\|\mathbf{w}_{k}\right\|^{2}\right\} \\
& =\frac{\beta_{\mathrm{SR}, j}-\sigma_{\mathrm{SR}, j}^{2}}{(M-K) \sigma_{\mathrm{SR}, k}^{2}} .
\end{aligned}
$$

Thus, we arrive at

$$
\mathrm{MI}_{k}=(1-\theta) P_{\mathrm{S}} \sum_{j \neq k}^{K} \frac{\beta_{\mathrm{SR}, j}-\sigma_{\mathrm{SR}, j}^{2}}{(M-K) \sigma_{\mathrm{SR}, k}^{2}} .
$$


(iv) Compute $\mathrm{ELI}_{k}$. From (31), the ELI associated with the ZF receiver can be expressed as

$$
\begin{aligned}
\mathrm{ELI}_{k} & =(1-\theta) P_{\mathrm{D}} \mathbb{E}\left\{\mathbf{w}_{k}^{H} \mathbf{G}_{\mathrm{DR}} \mathbf{G}_{\mathrm{DR}}^{H} \mathbf{w}_{k}\right\} \\
& =(1-\theta) P_{\mathrm{D}} \mathbb{E}\left\{\mathbf{w}_{k}^{H} \mathbf{D}_{\mathrm{DR}} \mathbf{w}_{k}\right\} \\
& =(1-\theta) P_{\mathrm{D}} \mathbb{E}\left\{\left\|\mathbf{w}_{k}\right\|^{2}\right\} \operatorname{Tr}\left\{\mathbf{D}_{\mathrm{DR}}\right\} \\
& =\frac{(1-\theta) P_{\mathrm{D}}}{(M-K) \sigma_{\mathrm{SR}, k}^{2}} \sum_{j=1}^{K} \beta_{\mathrm{DR}, j} .
\end{aligned}
$$

(v) Compute $A N_{k}$. Similarly, we obtain

$$
\mathrm{AN}_{k}=\frac{\sigma_{\mathrm{R}}^{2}}{(M-K) \sigma_{\mathrm{SR}, k}^{2}}
$$

Substituting (D.3), (D.4), (D.6), (D.7), and (D.8) into (29), we obtain

$$
\begin{aligned}
R_{\mathrm{SR}, k} & =\log _{2}(1 \\
& \left.+\frac{(M-K)(1-\theta) P_{\mathrm{S}} \sigma_{\mathrm{SR}, k}^{2}}{(1-\theta) \sum_{j=1}^{K}\left(P_{\mathrm{S}}\left(\beta_{\mathrm{SR}, j}-\sigma_{\mathrm{SR}, j}^{2}\right)+P_{\mathrm{D}} \beta_{\mathrm{DR}, j}\right)+\sigma_{\mathrm{R}}^{2}}\right) .
\end{aligned}
$$

Over i.i.d. fading, the achievable rate of the transmission link $\mathrm{S}_{k} \rightarrow \mathrm{R}$ can be expressed as

$$
\begin{aligned}
R_{\mathrm{SR}, k} & =\log _{2}(1 \\
& \left.+\frac{(M-K)(1-\theta) P_{\mathrm{S}} \sigma_{\mathrm{SR}}^{2}}{K(1-\theta)\left(P_{\mathrm{S}}\left(\beta_{\mathrm{SR}}-\sigma_{\mathrm{SR}}^{2}\right)+P_{\mathrm{D}} \beta_{\mathrm{DR}}\right)+\sigma_{\mathrm{R}}^{2}}\right) .
\end{aligned}
$$

(2) Derive $R_{D R, k}$. From (33), $\mathbb{E}\left\{\mathbf{g}_{\mathrm{DR}, k}^{T} \mathbf{a}_{k}\right\}$, $\operatorname{Var}\left(\mathbf{g}_{\mathrm{DR}, k}^{T} \mathbf{a}_{k}\right)$, and $\mathbb{E}\left\{\left|\mathbf{g}_{\mathrm{DR}, k}^{T} \mathbf{a}_{j}\right|^{2}\right\}$ are needed in computing $R_{\mathrm{RD}, k}$. By applying the procedures similarly to those in deriving $R_{\mathrm{SR}, k}$, we obtain

$$
\begin{aligned}
\mathbb{E}\left\{\mathbf{g}_{\mathrm{DR}, k}^{T} \mathbf{a}_{k}\right\} & =\alpha_{\mathrm{ZF}} \\
\operatorname{Var}\left(\mathbf{g}_{\mathrm{DR}, k}^{T} \mathbf{a}_{k}\right) & =\frac{\alpha_{\mathrm{ZF}}^{2}\left(\beta_{\mathrm{DR}, k}-\sigma_{\mathrm{DR}, k}^{2}\right)}{\sigma_{\mathrm{DR}, k}^{2}(M-K)} \\
\mathbb{E}\left\{\left|\mathbf{g}_{\mathrm{DR}, k}^{T} \mathbf{a}_{j}\right|^{2}\right\} & =\frac{\alpha_{\mathrm{ZF}}^{2}\left(\beta_{\mathrm{DR}, k}-\sigma_{\mathrm{DR}, k}^{2}\right)}{\sigma_{\mathrm{DR}, j}^{2}(M-K)}, \text { for } j \neq k .
\end{aligned}
$$

Substituting (D.11) into (33), we obtain

$$
\begin{aligned}
& R_{\mathrm{RD}, k}=\log _{2}(1 \\
& \left.+\frac{\alpha_{\mathrm{ZF}}^{2} p_{\mathrm{R}}}{\left(\alpha_{\mathrm{ZF}}^{2} p_{\mathrm{R}}\left(\beta_{\mathrm{DR}, k}-\sigma_{\mathrm{DR}, k}^{2}\right) /(M-K)\right) \sum_{j=1}^{K} \sigma_{\mathrm{DR}, j}^{-2}+\sigma_{\mathrm{D}}^{2}}\right) .
\end{aligned}
$$

Over i.i.d. fading, the achievable rate of the transmission link $\mathrm{R} \rightarrow \mathrm{D}_{k}$ can be expressed as

$$
\begin{aligned}
& R_{\mathrm{RD}, k}=\log _{2}(1 \\
& \left.+\frac{(M-K) \alpha_{\mathrm{ZF}}^{2} p_{\mathrm{R}}}{K \alpha_{\mathrm{ZF}}^{2} \sigma_{\mathrm{RD}}^{-2} p_{\mathrm{R}}\left(\beta_{\mathrm{RD}}-\sigma_{\mathrm{RD}}^{2}\right)+(M-K) \sigma_{\mathrm{D}}^{2}}\right),
\end{aligned}
$$

where the asymptotic expressions for $\alpha_{\mathrm{ZF}}$ and $P_{\mathrm{R}}$ over i.i.d. fading are, respectively, given by

$$
\begin{aligned}
& \alpha_{\mathrm{ZF}}^{2} \stackrel{\text { a.s. }}{\longrightarrow} \frac{M-K}{K \sigma_{\mathrm{RD}}^{-2}}, \\
& P_{\mathrm{R}} \stackrel{\text { a.s. }}{\longrightarrow} \eta \theta K\left(E_{\mathrm{S}} \beta_{\mathrm{SR}}+E_{\mathrm{D}} \beta_{\mathrm{DR}}\right) .
\end{aligned}
$$

Then, substituting (D.10) and (D.13) into $R_{\text {sum }}=$ $\sum_{k=1}^{K} \min \left\{R_{\mathrm{SR}, k}, R_{\mathrm{RD}, k}\right\}$, we arrive at (34).

\section{E. Proof of Proposition 9}

The SINRs $\gamma_{\mathrm{SR}}$ and $\gamma_{\mathrm{RD}}$ in (35) can be rewritten as

$$
\begin{aligned}
& \gamma_{\mathrm{SR}}=\frac{1}{a_{1} E_{\mathrm{ZF}}+b_{1}}, \\
& \gamma_{\mathrm{RD}}=\frac{E_{\mathrm{ZF}}}{c_{1} E_{\mathrm{ZF}}+d_{1}},
\end{aligned}
$$

respectively, where $E_{\mathrm{ZF}} \triangleq E_{\mathrm{S}} \beta_{\mathrm{SR}}+E_{\mathrm{D}} \beta_{\mathrm{RD}}, a_{1} \triangleq K /(M-$ $K) E_{\mathrm{S}} \sigma_{\mathrm{SR}}^{2}, b_{1} \triangleq M \sigma_{\mathrm{R}}^{2} /(M-K)(1-\theta) E_{\mathrm{S}} \sigma_{\mathrm{SR}}^{2}-K /(M-K)$, $c_{1} \triangleq K \sigma_{\mathrm{DR}}^{-2}\left(\beta_{\mathrm{DR}}-\sigma_{\mathrm{DR}}^{2}\right) /(M-K)$, and $d_{1} \triangleq \sigma_{\mathrm{D}}^{2} \sigma_{\mathrm{DR}}^{-2} / \eta \theta(M-K)$. From the point of view of practice, we assume that the CSI estimation error is small enough so that $\beta_{\mathrm{DR}}>\sigma_{\mathrm{DR}}^{2}$ and $c_{1}>0$ hold true. Since $E_{\mathrm{ZF}}$ is a linear transformation of $E_{\mathrm{D}}$, we first search the optimal $E_{\mathrm{ZF}}$ instead of the optimal $E_{\mathrm{D}}$. Note that $\gamma_{\mathrm{SR}}$ and $\gamma_{\mathrm{RD}}$ are decreasing and increasing functions with respect to $E_{\mathrm{D}} \in[0, \infty)$, respectively; we consider two cases of $\left.\gamma_{\mathrm{SR}}\right|_{E_{\mathrm{D}}=0} \leq\left.\gamma_{\mathrm{RD}}\right|_{E_{\mathrm{D}}=0}$ and $\left.\gamma_{\mathrm{SR}}\right|_{E_{\mathrm{D}}=0}>\left.\gamma_{\mathrm{RD}}\right|_{E_{\mathrm{D}}=0}$, respectively, in determining the optimal solution.

For the case of $\left.\gamma_{\mathrm{SR}}\right|_{E_{\mathrm{D}}=0} \leq\left.\gamma_{\mathrm{RD}}\right|_{E_{\mathrm{D}}=0}$, we have $E_{\mathrm{S}} \geq E_{\mathrm{ZF}} / \beta_{\mathrm{SR}}$ by reducing $\left.\gamma_{\mathrm{SR}}\right|_{E_{\mathrm{D}}=0} \leq\left.\gamma_{\mathrm{RD}}\right|_{E_{\mathrm{D}}=0}$. In such a case, we have $\gamma \triangleq$ $\min \left(\gamma_{\mathrm{SR}}, \gamma_{\mathrm{RD}}\right)=\gamma_{\mathrm{SR}}$ based on the monotonicities of $\gamma_{\mathrm{SR}}$ and $\gamma_{\mathrm{RD}}$. Since $\gamma_{\mathrm{SR}}$ is decreasing with respect to $E_{\mathrm{ZF}}$ (and $E_{\mathrm{D}}$ ), the optimal $E_{\mathrm{ZF}}$ that achieves the allowable maximum asymptotic sum rate as well as $\gamma$ is obtained by the allowable minimum $E_{\mathrm{ZF}}$, that is, by setting $E_{\mathrm{D}}=0$.

For the case of $\left.\gamma_{\mathrm{SR}}\right|_{E_{\mathrm{D}}=0}>\left.\gamma_{\mathrm{RD}}\right|_{E_{\mathrm{D}}=0}$, we have $E_{\mathrm{S}}>E_{\mathrm{ZF}} / \beta_{\mathrm{SR}}$ by reducing $\left.\gamma_{\mathrm{SR}}\right|_{E_{\mathrm{D}}=0} \leq\left.\gamma_{\mathrm{RD}}\right|_{E_{\mathrm{D}}=0}$. In such a case, $\gamma_{\mathrm{SR}} \geq \gamma_{\mathrm{RD}}$ for $E_{\mathrm{D}} \in\left(0, E_{\mathrm{D}}^{\mathrm{ZF}}\right]$ and $\gamma_{\mathrm{SR}}<\gamma_{\mathrm{RD}}$ for $E_{\mathrm{D}} \in\left(E_{\mathrm{D}}^{\mathrm{ZF}}, \infty\right)$, where $E_{\mathrm{D}}^{\mathrm{ZF}}$ is the root of $\gamma_{\mathrm{SR}}-\gamma_{\mathrm{RD}}=0$ with respect to $E_{\mathrm{D}}$. Thus, the allowable maximum asymptotic sum rate as well as $\gamma$ is obtained by $E_{\mathrm{D}}^{\mathrm{ZF}}$, which can be expressed as

$$
E_{\mathrm{D}}^{\mathrm{ZF}}=\frac{E_{\mathrm{ZF}}-E_{\mathrm{S}} \beta_{\mathrm{SR}}}{\beta_{\mathrm{DR}}}
$$

with $E_{\mathrm{ZF}} \triangleq\left(-b_{1}+c_{1}+\sqrt{\left(b_{1}-c_{1}\right)^{2}+4 a_{1} d_{1}}\right) / 2 a_{1}$ by solving $\gamma_{\mathrm{SR}}-\gamma_{\mathrm{RD}}=0$. This proves (38). 
Since the logarithm term $\log _{2}(1+\gamma)$ inherits the convexity/concavity of $\gamma$, determining the convexity/concavity of (35) with respect to $E_{\mathrm{D}}$ is equivalent to determining the convexity/concavity of $\gamma=\min \left(\gamma_{\mathrm{SR}}, \gamma_{\mathrm{RD}}\right)$ with respect to $E_{\mathrm{D}}$. For the case of $\left.\gamma_{\mathrm{SR}}\right|_{E_{\mathrm{D}}=0}>\left.\gamma_{\mathrm{RD}}\right|_{E_{\mathrm{D}}=0}$, it can be shown that $\gamma_{\mathrm{RD}}$ is concave with respect to $E_{\mathrm{D}} \in\left(0, E_{\mathrm{D}}^{\mathrm{ZF}}\right]$ and $\gamma_{\mathrm{SR}}$ is convex with respect to $E_{\mathrm{D}} \in\left(E_{\mathrm{D}}^{\mathrm{ZF}}, \infty\right)$. Thus, $\gamma=\min \left(\gamma_{\mathrm{SR}}, \gamma_{\mathrm{RD}}\right)$ as well as the asymptotic sum rate is neither convex nor concave for such a case.

\section{F. Proof of Proposition 10}

The SINRs $\gamma_{\mathrm{SR}}$ and $\gamma_{\mathrm{RD}}$ in (35) can be rewritten as

$$
\begin{aligned}
\gamma_{\mathrm{SR}} & =\frac{1-\theta}{a_{2}(1-\theta)+b_{2}}, \\
\gamma_{\mathrm{RD}} & =\frac{\theta}{c_{2} \theta+d_{2}},
\end{aligned}
$$

respectively, where $a_{2} \triangleq K\left(E_{\mathrm{S}}\left(\beta_{\mathrm{SR}}-\sigma_{\mathrm{SR}}^{2}\right)+E_{\mathrm{D}} \beta_{\mathrm{DR}}\right) /(M-$ $K) E_{\mathrm{S}} \sigma_{\mathrm{SR}}^{2}, b_{2} \triangleq M \sigma_{\mathrm{R}}^{2} /(M-K) E_{\mathrm{S}} \sigma_{\mathrm{SR}}^{2}, c_{2} \triangleq K \sigma_{\mathrm{DR}}^{-2}\left(\beta_{\mathrm{DR}}-\right.$ $\left.\sigma_{\mathrm{DR}}^{2}\right) /(M-K)$, and $d_{2} \triangleq \sigma_{\mathrm{D}}^{2} \sigma_{\mathrm{DR}}^{-2} / \eta(M-K)\left(E_{\mathrm{S}} \beta_{\mathrm{SR}}+E_{\mathrm{D}} \beta_{\mathrm{DR}}\right)$. From the point of view of practice, we assume that the CSI estimation error is small enough so that $\beta_{\mathrm{SR}}>\sigma_{\mathrm{SR}}^{2}, \beta_{\mathrm{DR}}>$ $\sigma_{\mathrm{DR}}^{2}, a_{2}>0$, and $c_{2}>0$ hold true. Since the allowable maximum asymptotic sum rate is achieved with the allowable maximum $\gamma=\min \left(\gamma_{\mathrm{SR}, \mathrm{RD}}\right)$, we continue the proof by searching $\theta$ that maximizes $\gamma$.

It can be shown that $\gamma_{\mathrm{SR}}$ and $\gamma_{\mathrm{RD}}$ are decreasing and increasing functions with respect to $\theta$, respectively. Furthermore, we have $\left.\gamma_{\mathrm{SR}}\right|_{\theta=0}>\left.\gamma_{\mathrm{RD}}\right|_{\theta=0}$ and $\left.\gamma_{\mathrm{SR}}\right|_{\theta=1}<\left.\gamma_{\mathrm{RD}}\right|_{\theta=1}$. Thus, there is a single cross-point between $\gamma_{\mathrm{SR}}$ and $\gamma_{\mathrm{RD}}$ for $\theta \in(0,1)$. Denote the cross-point by $\theta^{\mathrm{ZF}}$; it can be shown that $\gamma=\gamma_{\mathrm{RD}}$ for $\theta \in\left(0, \theta^{\mathrm{ZF}}\right)$ and $\gamma=\gamma_{\mathrm{SR}}$ for $\theta \in\left(\theta^{\mathrm{ZF}}, 1\right)$. Since $\gamma_{\mathrm{SR}}$ and $\gamma_{\mathrm{RD}}$ are concave with respect to $\theta \in(0,1)$, it can be concluded that $\gamma$ is concave with respect to $\theta$. Furthermore, noting the monotonicities of $\gamma_{\mathrm{SR}}$ and $\gamma_{\mathrm{RD}}$, the allowable maximum $\gamma$ is achieved by the root of $\gamma_{\mathrm{SR}}-\gamma_{\mathrm{RD}}=0$, which can be expressed as (39). This proves Proposition 10.

\section{G. Proof of Theorem 11}

We first prove that the asymptotic sum rate is neither convex nor concave with respect to $\left(\theta, E_{\mathrm{D}}\right)$. Since logarithm does not change convexity/concavity, determining the convexity/concavity of (35) with respect to $\left(\theta, E_{\mathrm{D}}\right)$ is equivalent to determining the convexity/concavity of $\gamma \triangleq \min \left(\gamma_{\mathrm{SR}}, \gamma_{\mathrm{RD}}\right)$ with respect to $\left(\theta, E_{\mathrm{D}}\right)$. It can be shown that $\gamma=\gamma_{\mathrm{SR}}$ exists with a nonzero probability, so that we choose to investigate the convexity/concavity of $\gamma_{\mathrm{SR}}$ without loss of generality. The expression of $\gamma_{\mathrm{SR}}$ in (36) can be rewritten as

$$
\gamma_{\mathrm{SR}}=\frac{1-\theta}{a(1-\theta) E_{\mathrm{D}}+b},
$$

where $a \triangleq K \beta_{\mathrm{RD}} /(M-K) E_{\mathrm{S}} \sigma_{\mathrm{SR}}^{2}$ and $b \triangleq\left(K E_{\mathrm{S}}\left(\beta_{\mathrm{SR}}-\sigma_{\mathrm{SR}}^{2}\right)+\right.$ $\left.M \sigma_{\mathrm{R}}^{2}\right) /(M-K) E_{\mathrm{S}} \sigma_{\mathrm{SR}}^{2}$. From the point of view of practice, we assume that the CSI estimation error is small enough so that $\beta_{\mathrm{SR}}>\sigma_{\mathrm{SR}}^{2}$ and $b>0$ hold true. Then, the Hessian of $\gamma_{\mathrm{SR}}$ with respect to $\left(\theta, E_{\mathrm{D}}\right)$ can be expressed as

$$
\widetilde{H}=\left[\begin{array}{cc}
-\frac{2 a b E_{\mathrm{D}}}{\left(b+a(1-\theta) E_{\mathrm{D}}\right)^{3}} & \frac{2 a b(1-\theta)}{\left(b+a(1-\theta) E_{\mathrm{D}}\right)^{3}} \\
\frac{2 a b(1-\theta)}{\left(b+a(1-\theta) E_{\mathrm{D}}\right)^{3}} & \frac{2 a^{2}(1-\theta)^{3}}{\left(b+a(1-\theta) E_{\mathrm{D}}\right)^{3}}
\end{array}\right] .
$$

Since $\partial^{2} \gamma_{\mathrm{SR}} / \partial \theta^{2}=-2 a b E_{\mathrm{D}} /\left(b+a(1-\theta) E_{\mathrm{D}}\right)^{3}<0$ and $|\widetilde{H}|<0$, $\widetilde{H}$ is neither positive definite nor negative definite. Therefore, $\gamma_{\mathrm{SR}}$ is neither convex nor concave with respect to $\left(\theta, E_{\mathrm{D}}\right)$.

For the proof of the uniqueness of the optimal $\left(\theta, E_{\mathrm{D}}\right)$, we proceed by contradiction. Denote $\left(\theta^{*}, E_{\mathrm{D}}^{*}\right)$ as an optimal point that achieves the maximum asymptotic sum rate. From Proposition 9, we know that $E_{\mathrm{D}}^{*}=E_{\mathrm{D}}^{\mathrm{ZF}}\left(\theta^{*}\right)$ is uniquely determined so that

$$
R_{\text {sum }}\left(\theta^{*}, E_{\mathrm{D}}^{*}\right)=R_{\text {sum }}\left(\theta^{*}, E_{\mathrm{D}}^{\mathrm{ZF}}\left(\theta^{*}\right)\right) .
$$

From Proposition 10, we know that $\theta^{*}=\theta^{z F}\left(E_{D}^{*}\right)$ is uniquely determined so that

$$
R_{\text {sum }}\left(\theta^{*}, E_{\mathrm{D}}^{*}\right)=R_{\text {sum }}\left(\theta^{\mathrm{ZF}}\left(E_{\mathrm{D}}^{*}\right), E_{\mathrm{D}}^{*}\right) .
$$

Suppose that there is another optimal point $\left(\widetilde{\theta}^{*}, \widetilde{E}_{\mathrm{D}}^{*}\right)$ that achieves the maximum sum rate with $\widetilde{\theta}^{*} \neq \theta^{*}$ and $\widetilde{E}_{\mathrm{D}}^{*} \neq E_{\mathrm{D}}^{*}$. From Proposition 9, we have

$$
R_{\text {sum }}\left(\theta^{*}, E_{\mathrm{D}}^{\mathrm{ZF}}\left(\widetilde{\theta}^{*}\right)\right)<R_{\text {sum }}\left(\theta^{*}, E_{\mathrm{D}}^{\mathrm{ZF}}\left(\theta^{*}\right)\right) .
$$

Since $R_{\text {sum }}$ is concave with respect to $\theta$, for $\theta^{*}$ that achieves the maximum $R_{\text {sum }}$ with any given destination transmission power, we have

$$
R_{\text {sum }}\left(\widetilde{\theta}^{*}, E_{\mathrm{D}}^{\mathrm{ZF}}\left(\widetilde{\theta}^{*}\right)\right)<R_{\text {sum }}\left(\theta^{*}, E_{\mathrm{D}}^{\mathrm{ZF}}\left(\widetilde{\theta}^{*}\right)\right)
$$

with $\widetilde{\theta}^{*} \neq \theta^{*}$. With (G.5) and (G.6), we have

$$
\begin{aligned}
R_{\text {sum }}\left(\widetilde{\theta}^{*}, \widetilde{E}_{\mathrm{D}}^{*}\right) & =R_{\text {sum }}\left(\widetilde{\theta}^{*}, E_{\mathrm{D}}^{\mathrm{ZF}}\left(\widetilde{\theta}^{*}\right)\right) \\
& <R_{\text {sum }}\left(\theta^{*}, E_{\mathrm{D}}^{\mathrm{ZF}}\left(\widetilde{\theta}^{*}\right)\right) \\
& <R_{\text {sum }}\left(\theta^{*}, E_{\mathrm{D}}^{\mathrm{ZF}}\left(\theta^{*}\right)\right) \\
& =R_{\text {sum }}\left(\theta^{*}, E_{\mathrm{D}}^{*}\right),
\end{aligned}
$$

which contradicts the fact that $\left(\widetilde{\theta}^{*}, \widetilde{E}_{\mathrm{D}}^{*}\right)$ is an optimal point.

\section{H. Proof of Theorem 12}

First, we derive $R_{\mathrm{SR}, k}$. Since $\widehat{\mathbf{g}}_{\mathrm{SR}, k}^{H} \mathbf{g}_{\mathrm{SR}, k}=\left\|\mathbf{g}_{\mathrm{SR}, k}\right\|^{2}+\widehat{\mathbf{g}}_{\mathrm{SR}, k} \mathscr{E}_{\mathrm{SR}, k}$, it can be shown that

$$
\mathbb{E}\left\{\widehat{\mathbf{g}}_{\mathrm{SR}, k}^{H} \mathbf{g}_{\mathrm{SR}, k}\right\}=\mathbb{E}\left\{\left\|\mathbf{g}_{\mathrm{SR}, k}\right\|^{2}\right\}=\sigma_{\mathrm{SR}, k}^{2} M .
$$

With the help of [25, Lemma 2.9], the variance of $\widehat{\mathbf{g}}_{\mathrm{SR}, k}^{H} \mathbf{g}_{\mathrm{SR}, k}$ can be expressed as

$$
\operatorname{Var}\left(\widehat{\mathbf{g}}_{\mathrm{SR}, k}^{H} \mathbf{g}_{\mathrm{SR}, k}\right)=\sigma_{\mathrm{SR}, k}^{2} \beta_{\mathrm{SR}, k} M .
$$


Similarly, we have

$$
\begin{aligned}
\mathrm{MI}_{k} & =(1-\theta) P_{\mathrm{S}} \sigma_{\mathrm{SR}, k}^{2} M \sum_{j \neq k}^{K} \beta_{\mathrm{SR}, j}, \\
\mathrm{ELI}_{k} & =(1-\theta) P_{\mathrm{D}} \sigma_{\mathrm{SR}, k}^{2} M \sum_{j=1}^{K} \beta_{\mathrm{RD}, j}, \\
\mathrm{AN}_{k} & =\sigma_{\mathrm{R}}^{2} \sigma_{\mathrm{SR}, k}^{2} M .
\end{aligned}
$$

By substituting (H.1), (H.2), and (H.3) into (29), we can obtain

$$
\begin{aligned}
R_{\mathrm{SR}, k} & =\log _{2}(1 \\
+ & \left.\frac{(1-\theta) P_{\mathrm{S}} \sigma_{\mathrm{SR}, k}^{2} M}{(1-\theta) \sum_{j=1}^{K}\left(P_{\mathrm{S}} \beta_{\mathrm{SR}, j}+P_{\mathrm{D}} \beta_{\mathrm{RD}, j}\right)+\sigma_{\mathrm{R}}^{2}}\right) .
\end{aligned}
$$

Over i.i.d. fading, the achievable rate of the transmission link $\mathrm{S}_{k} \rightarrow \mathrm{R}$ can be expressed as

$$
\begin{aligned}
& R_{\mathrm{SR}, k} \\
& \quad=\log _{2}\left(1+\frac{(1-\theta) P_{\mathrm{S}} \sigma_{\mathrm{SR}, k}^{2} M}{K(1-\theta)\left(P_{\mathrm{S}} \beta_{\mathrm{SR}}+P_{\mathrm{D}} \beta_{\mathrm{RD}}\right)+\sigma_{\mathrm{R}}^{2}}\right),
\end{aligned}
$$

where $\sigma_{\mathrm{SR}}^{2} \triangleq \tau_{\mathrm{p}} P_{\mathrm{p}} \beta_{\mathrm{SR}}^{2} /\left(\tau_{\mathrm{p}} P_{\mathrm{p}} \beta_{\mathrm{SR}}+\sigma_{\mathrm{R}, \mathrm{p}}^{2}\right)$. Similarly, we arrive at (42).

\section{Competing Interests}

The authors declare that there are no competing interests regarding the publication of this paper.

\section{Acknowledgments}

This work was supported in part by SRF for ROCS, SEM, Shandong Provincial Natural Science Foundation, China, under Grant 2014ZRB019XM, in part by the Ministry of Science, ICT and Future Planning (MSIP), Korea, under the Information Technology Research Center Support Program supervised by the Institute for Information and Communications Technology Promotion under Grant IITP-2016-H850116-1019, and in part by the National Research Foundation of Korea, Grant Funded by the Korean Government, MSIP, under Grant NRF-2014K1A3A1A20034987.

\section{References}

[1] A. A. Nasir, X. Zhou, S. Durrani, and R. A. Kennedy, "Relaying protocols for wireless energy harvesting and information processing," IEEE Transactions on Wireless Communications, vol.12, no. 7, pp. 3622-3636, 2013.

[2] H. Ju and R. Zhang, "User cooperation in wireless powered communication networks," in Proceedings of the IEEE Global Communications Conference (GLOBECOM '14), pp. 1430-1435, IEEE, Austin, Tex, USA, December 2014.
[3] Y. Huang and B. Clerckx, "Joint wireless information and power transfer for an autonomous multiple antenna relay system," IEEE Communications Letters, vol. 19, no. 7, pp. 1113-1116, 2015.

[4] R. Sun, Y. Wang, Z. Miao, and X. Wang, "Destination-aided wireless power transfer in energy-limited cognitive relay systems," IEEE Access, vol. 4, pp. 5385-5398, 2016.

[5] Z. Ding, S. M. Perlaza, I. Esnaola, and H. V. Poor, "Power allocation strategies in energy harvesting wireless cooperative networks," IEEE Transactions on Wireless Communications, vol. 13, no. 2, pp. 846-860, 2014.

[6] I. Krikidis, "Simultaneous information and energy transfer in large-scale networks with/without relaying," IEEE Transactions on Communications, vol. 62, no. 3, pp. 900-912, 2014.

[7] H. Chen, Y. Li, Y. Jiang, Y. Ma, and B. Vucetic, "Distributed power splitting for SWIPT in relay interference channels using game theory," IEEE Transactions on Wireless Communications, vol. 14, no. 1, pp. 410-420, 2015.

[8] A. A. Nasir, D. T. Ngo, X. Zhou, R. A. Kennedy, and S. Durrani, "Joint resource optimization for multicell networks with wireless energy harvesting relays," IEEE Transactions on Vehicular Technology, vol. 65, no. 8, pp. 6168-6183, 2016.

[9] Z. Chang, J. Gong, Y. Li et al., "Energy efficient resource allocation for wireless power transfer enabled collaborative mobile clouds," IEEE Journal on Selected Areas in Communications, vol. 34, no. 12, pp. 3438-3450, 2016.

[10] H. Liu and K. S. Kwak, "Multipair massive MIMO relay with simultaneous wireless information and power transfer," in Proceedings of the International Conference on Information and Communication Technology Convergence (ICTC '16), pp. 225230, Jeju Island, Korea, October 2016.

[11] Z. Ding, C. Zhong, D. W. K. Ng et al., "Application of smart antenna technologies in simultaneous wireless information and power transfer," IEEE Communications Magazine, vol. 53, no. 4, pp. 86-93, 2015.

[12] R. Zhang and C. K. Ho, "MIMO broadcasting for simultaneous wireless information and power transfer," IEEE Transactions on Wireless Communications, vol. 12, no. 5, pp. 1989-2001, 2013.

[13] I. Krikidis, S. Sasaki, S. Timotheou, and Z. Ding, "A low complexity antenna switching for joint wireless information and energy transfer in MIMO relay channels," IEEE Transactions on Communications, vol. 62, no. 5, pp. 1577-1587, 2014.

[14] T. L. Marzetta, "Noncooperative cellular wireless with unlimited numbers of base station antennas," IEEE Transactions on Wireless Communications, vol. 9, no. 11, pp. 3590-3600, 2010.

[15] E. G. Larsson, O. Edfors, F. Tufvesson, and T. L. Marzetta, "Massive MIMO for next generation wireless systems," IEEE Communications Magazine, vol. 52, no. 2, pp. 186-195, 2014.

[16] L. Lu, G. Y. Li, A. L. Swindlehurst, A. Ashikhmin, and R. Zhang, "An overview of massive MIMO: benefits and challenges," IEEE Journal on Selected Topics in Signal Processing, vol. 8, no. 5, pp. 742-758, 2014

[17] H. Q. Ngo, H. A. Suraweerat, M. Matthaiou, and E. G. Larsson, "Multipair massive MIMO full-duplex relaying with MRC/ MRT processing," in Proceedings of the 1st IEEE International Conference on Communications (ICC '14), pp. 4807-4813, IEEE, Sydney, Australia, June 2014.

[18] X. Chen, Z. Zhang, H.-H. Chen, and H. Zhang, "Enhancing wireless information and power transfer by exploiting multiantenna techniques," IEEE Communications Magazine, vol. 53, no. 4, pp. 133-141, 2015. 
[19] G. Yang, C. K. Ho, R. Zhang, and Y. L. Guan, "Throughput optimization for massive MIMO systems powered by wireless energy transfer," IEEE Journal on Selected Areas in Communications, vol. 33, no. 8, pp. 1640-1650, 2015.

[20] H. Wang, W. Wang, X. Chen, and Z. Zhang, "Wireless information and energy transfer in interference aware massive MIMO systems," in Proceedings of the IEEE Global Communications Conference (GLOBECOM '14), pp. 2556-2561, IEEE, Austin, Tex, USA, December 2014.

[21] G. Amarasuriya and H. V. Poor, "Wireless information and power transfer in multi-way relay networks with massive MIMO," in Proceedings of the 58th IEEE Global Communications Conference (GLOBECOM '15), pp. 1-7, San Diego, Calif, USA, December 2015.

[22] T. Wang, A. Cano, G. B. Giannakis, and J. N. Laneman, "High-performance cooperative demodulation with decodeand-forward relays," IEEE Transactions on Communications, vol. 55, no. 7, pp. 1427-1438, 2007.

[23] H. Yang and T. L. Marzetta, "Performance of conjugate and zeroforcing beamforming in large-scale antenna systems," IEEE Journal on Selected Areas in Communications, vol. 31, no. 2, pp. 172-179, 2013.

[24] S. Boyd and L. Vandenberghe, Convex Optimization, Cambridge University Press, Cambridge, UK, 2004.

[25] A. M. Tulino and S. Verdú, Random Matrix Theory and Wireless Communications: Foundations and Trends in Communications and Information Theory, Now Publishers Inc, Hanover, Mass, USA, 2004. 

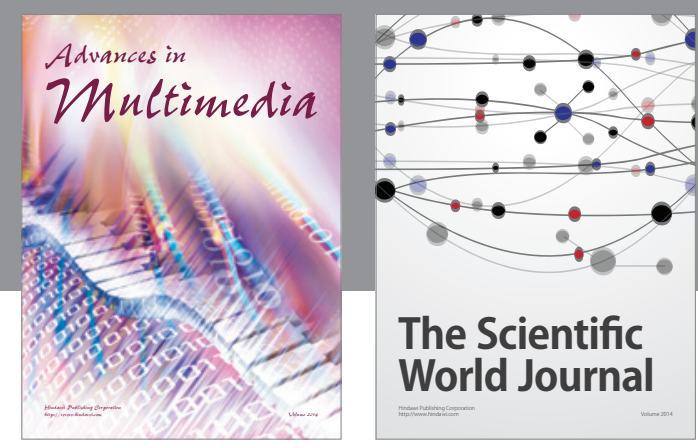

The Scientific World Journal
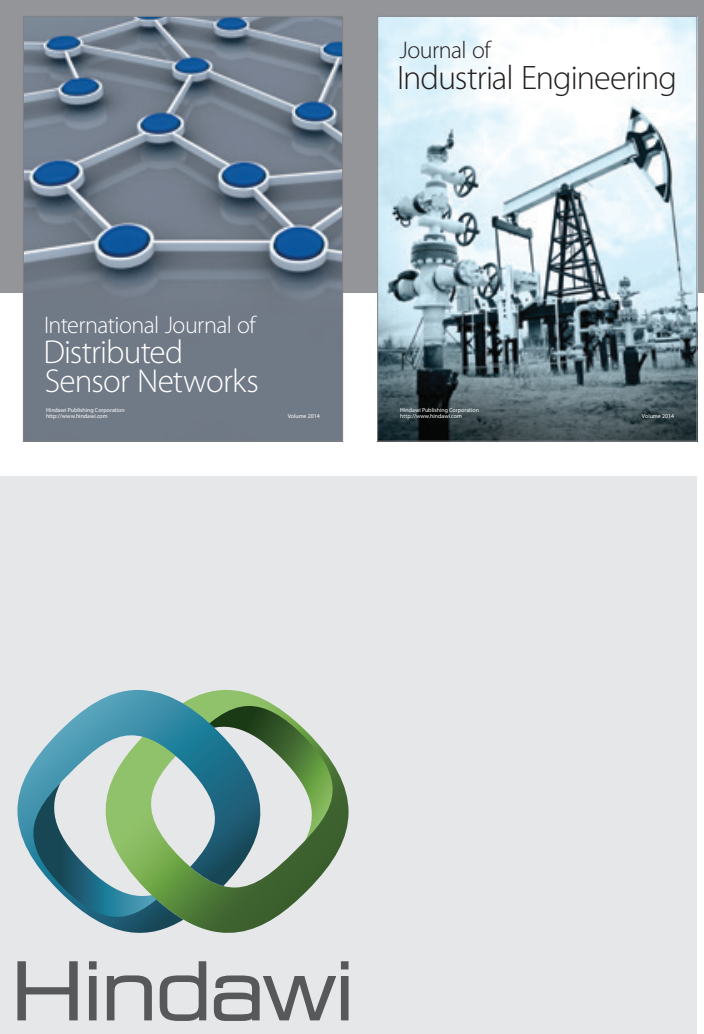

Submit your manuscripts at

https://www.hindawi.com

\section{Computer Networks} and Communications
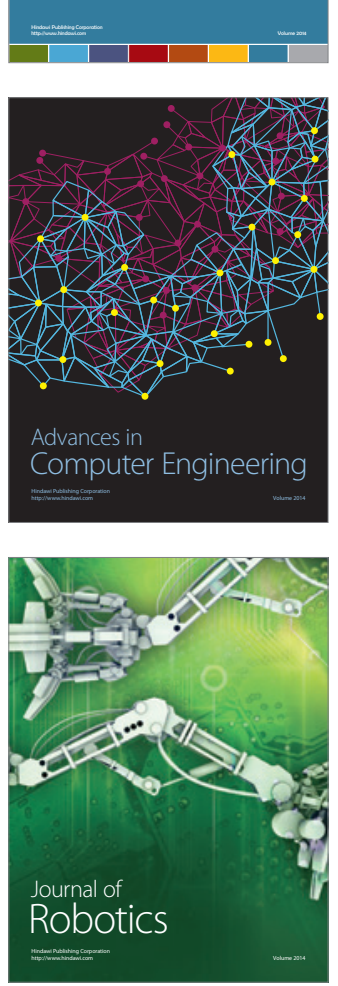
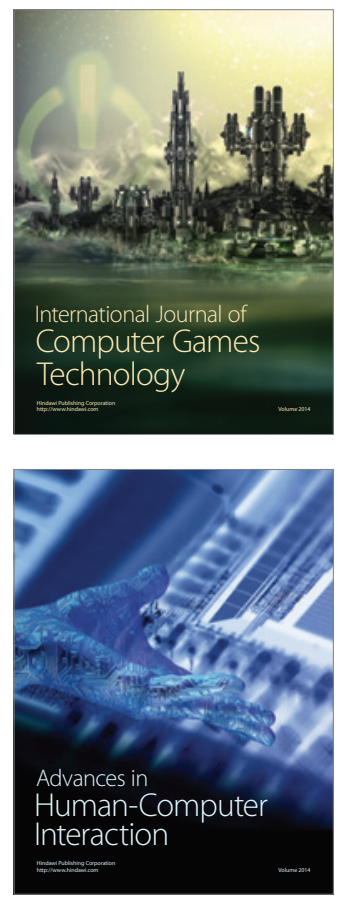
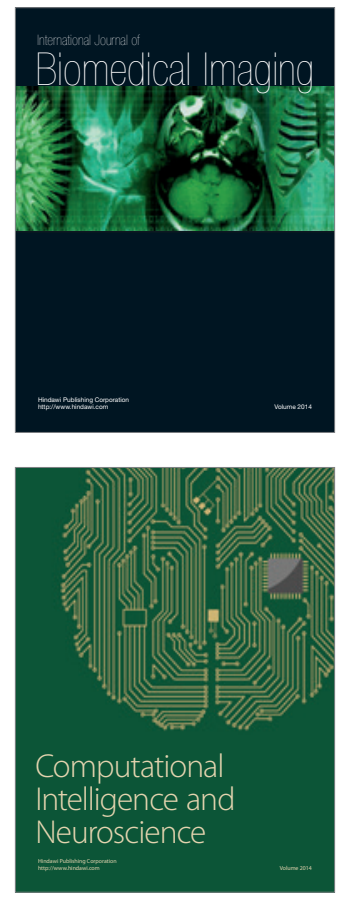
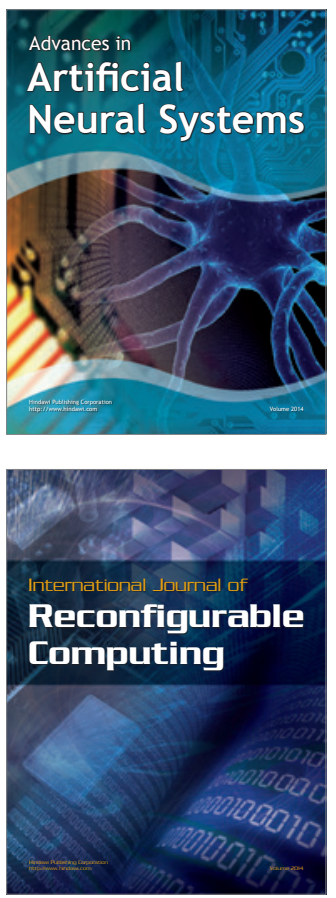
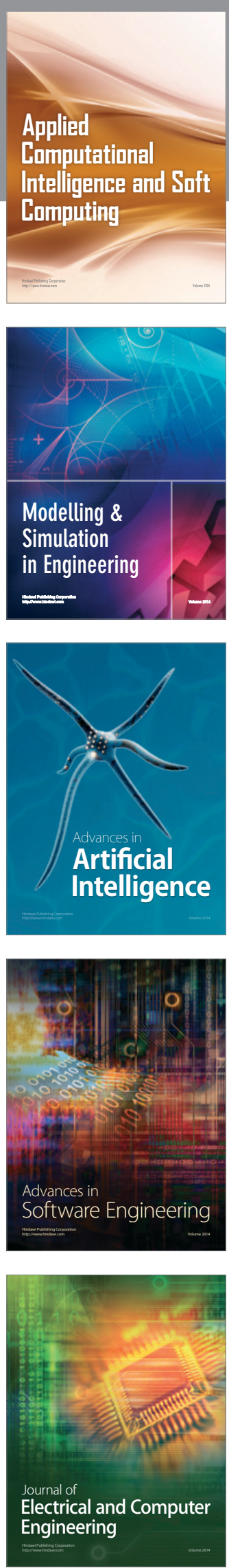